\title{
The Genetic Structure of Pseudoperonospora cubensis Populations
}

L. M. Quesada-Ocampo, Visiting Research Associate, L. L. Granke, Visiting Research Associate, J. Olsen, Undergraduate Research Assistant, and H. C. Gutting, Undergraduate Research Assistant, Department of Plant Pathology, Michigan State University, East Lansing 48824; F. Runge, Graduate Research Assistant, Institute of Botany 210, University of Hohenheim, D-70593 Stuttgart, Germany; M. Thines, Professor, Department of Biological Sciences, Institute of Ecology, Evolution and Diversity, Johann Wolfgang Goethe University; Biodiversity and Climate Research Centre (BiK-F); and Senckenberg Gesellschaft für Naturforschung, D-60325 Frankfurt (Main), Germany; A. Lebeda, Professor, Department of Botany, Palacky University in Olomouc, Slechtitelu 11, 783 71 OlomoucHolice, Czech Republic; and M. K. Hausbeck, Professor, Department of Plant Pathology, Michigan State University

\begin{abstract}
Quesada-Ocampo, L. M., Granke, L. L., Olsen, J., Gutting, H. C., Runge, F., Thines, M., Lebeda, A., and Hausbeck, M. K. 2012. The genetic structure of Pseudoperonospora cubensis populations. Plant Dis. 96:1459-1470.

Pseudoperonospora cubensis is a destructive foliar pathogen of economically important cucurbitaceous crops in the United States and worldwide. In this study, we investigated the genetic structure of $465 P$. cubensis isolates from three continents, 13 countries, 19 states of the United States, and five host species using five nuclear and two mitochondrial loci. Bayesian clustering resolved six genetic clusters and suggested some population structure by geographic origin and host, because some clusters occurred more or less frequently in particular categories. All of the genetic clusters were present in the sampling

were observed by country and state. Isolates from cucumber had different cluster composition and lower genetic diversity than isolates from other cucurbits. Because genetic structuring was detected, isolates that represent the genetic variation in $P$. cubensis should be used when developing diagnostic tools, fungicides, and resistant host varieties. Although this study provides an initial map of global population structure of $P$. cubensis, future genotyping of isolates could reveal population structure within specific geographic regions, across a wider range of hosts, or during different time points during the growing season.
\end{abstract} from North America and Europe. Differences in cluster occurrence
Pseudoperonospora cubensis (Berk. \& M.A. Curtis) Rostovzev is the causal agent of cucurbit downy mildew, a destructive foliar disease reported in more than 70 countries $(24,58)$. P. cubensis causes disease on approximately 20 genera of cucurbits (approximately 60 species ) including cucumber (Cucumis sativus), cantaloupe (C. melo), pumpkin (Cucurbita maxima), watermelon (Citrullus lanatus), squash (Cucurbita pepo), gourd (C. moschata), and several wild Cucurbitaceae species $(29,41,51,62)$. There may exist two distinct subspecies or species that parasitize cucurbits within the $P$. cubensis species complex (50) (P. cubensis sensu lato, which is referred to as $P$. cubensis in this article), and different pathotypes and physiological races in $P$. cubensis have been previously described $(24,55)$.

Cucurbit downy mildew has been a major pathogen in Europe since the mid-1980s (27), and severe disease pressure results in a reduction in quantity and quality of cucurbit fruit production (26). In the United States (U.S.), host resistance successfully limited downy mildew of cucumber for many years. Since 2004, resistant cucumber varieties are no longer sufficient to control $P$. cubensis in the U.S., and severe economic losses result if fungicides are not applied frequently (21). High costs associated with fungicide use and fungicide-resistant populations of $P$. cubensis that have been documented in several regions challenge the profitability of the industry $(34,36,46,53,63,64,67)$.

Because $P$. cubensis is an obligate parasite, its survival is dependent on the availability of a susceptible host (8). The pathogen overwinters (3) in areas with mild winter temperatures such as the southern U.S. and in greenhouses that produce cucurbits year-

Corresponding author: M. K. Hausbeck, E-mail: hausbec1@msu.edu

* The $\boldsymbol{e}$-Xtra logo stands for "electronic extra" and indicates that a Supplementary table is available online.

Accepted for publication 4 May 2012.

http://dx.doi.org/10.1094/PDIS-11-11-0943-RE

(C) 2012 The American Phytopathological Society round (61). Sporangia are primarily wind dispersed within and between fields (61), and P. cubensis spreads northward each growing season on northerly winds $(14,20,65)$. In the U.S., it is currently thought that $P$. cubensis overwinters in southern Florida, which provides a source of inoculum for states as far north as North Carolina. It has been hypothesized that the Great Lakes and midAtlantic states receive inoculum through dispersal of sporangia from North Carolina and surrounding states or from greenhouses that produce cucumber year-round in neighboring regions (40).

Populations of $P$. cubensis have been previously characterized for pathogenicity, virulence and fungicide resistance $(25,28)$, and populations have been studied using traditional phylogenetic methods and a single nuclear marker (nuclear internal transcribed spacer [nrITS]) in the Czech Republic, Greece, the Netherlands, and France, where this pathogen causes significant losses (54). Such investigations provided some information about local genetic diversity of P. cubensis. No genetic structure was noted by pathogenicity or virulence but isolates showed structure by geographic origin (54). It is currently unknown whether P. cubensis populations are structured by geography across continental scales or within the U.S., although Runge et al. (50) observed some spatiotemporal structure in their phylogenetic investigation.

Bayesian clustering has recently been used to visualize overall patterns of genetic structure in other oomycete $(18,44)$ and fungal $(5,16)$ pathogens. In this study, we used Bayesian clustering and genetic diversity and differentiation estimates to examine the genetic structure of worldwide $P$. cubensis sensu lato populations. Specifically, we sought to establish whether $P$. cubensis worldwide populations show significant population structure and genetic differentiation when grouped by geography and host of origin. We also examined population structure by year for isolates collected in the U.S. This study provides an initial map of the global population structure of $P$. cubensis.

\section{Materials and Methods}

Isolate selection and maintenance. In total, 465 P. cubensis isolates originating from diverse hosts throughout the world were obtained from colleagues or selected from the collection main- 
tained in the laboratory of Dr. Hausbeck at Michigan State University (MSU) (Supplementary Table S1). Samples included sporangial suspensions and individual leaf lesions that were used for DNA extractions. To obtain sporangial suspensions, $P$. cubensis sporangia were removed from leaves by pipetting sterile distilled water onto lesions on the leaf, dislodging sporangia using an ethanol-disinfested bacterial loop, and using a pipette to transfer the spore suspension from the leaf into a $2.2-\mathrm{ml}$ sterile microcentrifuge tube. When sporulation was not present on the leaf, lesions that displayed symptoms typical of downy mildew were excised with a sterile scalpel and placed into a 2.2-ml sterile microcentrifuge tube.

DNA extraction. Contents of microcentrifuge tubes with sporangia suspensions or leaf lesions were transferred to a mortar and ground with a pestle in liquid nitrogen. All implements were previously sterilized. The DNeasy Plant Minikit (Qiagen) was used to extract genomic DNA according to the manufacturer's instructions. DNA was quantified using the NanoDrop ND 1000 spectrophotometer and NanoDrop 2.4.7c software (NanoDrop Technologies Inc.). DNA integrity was analyzed by electrophoresis in $1 \%$ (wt/vol) agarose gel in $0.5 \times$ Tris-borate-EDTA buffer (32), and stained with ethidium bromide $(5 \mu \mathrm{g} / \mathrm{ml})$ for visualization.

Primer design, DNA amplification, and sequencing. GenBank was used to identify genomic regions as sources of single nucleotide polymorphism markers (Table 1). Selected regions were previously used in oomycete coalescence, genetic diversity, and phylogenetic studies $(7,13,17,42)$. Oomycete candidate genes were compared with the preliminary data of the $P$. cubensis genome project (courtesy B. Day, MSU) using BLAST (33). Sequences from GenBank and the $P$. cubensis genome were used as input data in Primer3 (49) for primer design using default settings. Two regions of the mitochondrial genome (subunit 1 and 5 of the NADH dehydrogenase gene [Nadl and Nad5, respectively) and five nuclear genes ( $\beta$-tubulin, glucose phosphate isomerase [gpi], nrITS, large ribosomal subunit [nrLSU], and TigA gene fusion protein $[$ TigA]) were amplified by polymerase chain reaction (PCR). After collecting preliminary sequence data, additional nested primers were designed for TigA to increase sequencing quality. The forward primer for nrITS (12) and both nrLSU primers $(35,47)$ were obtained from previously published literature. A $25-\mu \mathrm{l}$-volume PCR was executed using $1 \mu \mathrm{l}$ of DNA at $5 \mathrm{ng} / \mu \mathrm{l}, 5 \mu \mathrm{l}$ of $5 \times \mathrm{PCR}$ reaction buffer (Invitrogen), $1.25 \mu \mathrm{l}$ of $25 \mathrm{mM} \mathrm{MgCl}_{2}$ (Invitrogen), $0.5 \mu \mathrm{l}$ of $10 \mathrm{mM} \mathrm{dNTP}$ mix (Invitrogen), $1 \mu \mathrm{l}$ of each $10 \mu \mathrm{M}$ primer (MSU Macromolecular Structure Facility), $0.7 \mu$ of Platinum Taq DNA polymerase (Invitrogen), and $14.6 \mu \mathrm{l}$ of sterile water. A Mastercycler thermal cycler (Eppendorf) was used to run the PCR as follows: 3 min of denaturation at $94^{\circ} \mathrm{C}$; followed by 45 cycles at $94^{\circ} \mathrm{C}$ for $30 \mathrm{~s}$, annealing at $56^{\circ} \mathrm{C}$ for $30 \mathrm{~s}$, and extension at $72^{\circ} \mathrm{C}$ for $60 \mathrm{~s}$; with a final extension step of $10 \mathrm{~min}$ at $72^{\circ} \mathrm{C}$. PCR products were analyzed by electrophoresis in $1 \%$ (wt/vol) agarose gel in $0.5 \times$ Tris-borate-EDTA buffer (32), stained with ethidium bromide $(5 \mu \mathrm{g} / \mathrm{ml})$ for visualization, and compared with a 100-bp ladder (Invitrogen). Controls without $P$. cubensis DNA were included.

ExoSAP-IT (Affymetrix, Inc.) was used according to the manufacturer's protocol to purify PCR products, which were subse-

Table 1. Mitochondrial and nuclear genes (subunit $1[\mathrm{Nadl}]$ and 5 [Nad5] of the NADH dehydrogenase gene, $\beta$-tubulin, glucose phosphate isomerase [gpi], nuclear ribosomal internal transcribed spacer [nrITS], large nuclear ribosomal subunit [nrLSU], and TigA gene fusion protein [TigA]) analyzed in Pseudoperonospora cubensis

\begin{tabular}{|c|c|c|c|c|c|c|}
\hline Target DNA & Primer & Primer sequence & $\begin{array}{c}\text { Annealing } \\
\text { temperature }\left({ }^{\circ} \mathbf{C}\right)\end{array}$ & $\begin{array}{c}\text { Sequenced } \\
\text { fragment (bp) }\end{array}$ & $\begin{array}{l}\text { Analyzed } \\
\text { region (bp) }\end{array}$ & Source \\
\hline \multicolumn{7}{|l|}{ Mitochondrial } \\
\hline \multirow[t]{2}{*}{ Nadl } & Nad1-F & 5'TGGACGGTAATCCCTTTAGGA3' & 59 & 499 & 425 & DQ361222 \\
\hline & Nad2-R & 5'ATGGTGCTAACCATCCACCT3' & 59 & & & \\
\hline \multirow[t]{2}{*}{ Nad5 } & Nad5-F & 5'TGCAGCAACAATGGTAACAG3' & 58 & 320 & 255 & AY423338 \\
\hline & Nad5-R & 5'CTTGCTCATCACCCATTGC3' & 58 & $\ldots$ & $\ldots$ & $\ldots$ \\
\hline \multirow{3}{*}{$\begin{array}{l}\text { Nuclear } \\
\beta \text {-tubulin }\end{array}$} & & & & & & \\
\hline & BTub-F & 5'GTCGAAAATGCCGATGAAGT3' & 60 & 419 & 350 & DQ361162 \\
\hline & BTub-R & 5’TTCAGCATTTGCTCGTCAAC3' & 60 & & & \\
\hline \multirow[t]{2}{*}{ gpi } & GPI-F & 5'TGGTAAGCGTGTAAGCATGG3' & 59 & 430 & 365 & Draft genome \\
\hline & GPI-R & 5'GTTGATGCCCCAGATAGAGC3' & 59 & & & \\
\hline \multirow[t]{2}{*}{ nrITS } & ITS-F & 5'GAGGGACTTTTGGGTAATCA3' & 59 & 700 & 620 & 12 \\
\hline & ITS-R & 5'TCCTCCGCTTATTGATATGC3' & 59 & $\ldots$ & $\ldots$ & Draft genome \\
\hline \multirow[t]{2}{*}{ nrLSU } & LSU-F & 5'CGCCAGACGAGCTTACC 3' & 59 & 800 & 500 & 47 \\
\hline & LSU-R & 5'ACCCGCTGAACTTAAGC3' & 59 & $\ldots$ & $\ldots$ & 35 \\
\hline \multirow[t]{3}{*}{$\operatorname{TigA}$} & TigA-F & 5'GACATTGACGGATTCCTCGT3' & 59 & 511 & 400 & Draft genome \\
\hline & TigA-R & 5'CTCGTACAGCTCGTGGTTCA3' & 59 & $\ldots$ & $\ldots$ & $\ldots$ \\
\hline & TigA-R & 5'CGTTCACAACGATGTGCTTT3' & 60 & 298 & 200 & Sequenced product \\
\hline
\end{tabular}

Table 2. Diversity estimates, neutrality tests, and recombination for target mitochondrial and nuclear genes in Pseudoperonospora cubensis

\begin{tabular}{|c|c|c|c|c|c|c|c|c|c|c|c|}
\hline \multirow[b]{2}{*}{ Target DNA ${ }^{\mathrm{d}}$} & \multicolumn{6}{|c|}{ Diversity estimates $^{\mathbf{a}}$} & \multicolumn{2}{|c|}{$\begin{array}{c}\text { Recombination } \\
\text { estimates }^{\mathrm{b}}\end{array}$} & \multicolumn{3}{|c|}{ Neutrality tests ${ }^{\mathrm{c}}$} \\
\hline & s $(\%)$ & h & Hd & $\pi$ & $\theta w$ & $\mathbf{k}$ & $\mathbf{R}$ & $\mathbf{R M}$ & Tajima's D & Fu and Li's D & Fu and Li's F \\
\hline \multicolumn{12}{|l|}{ Mitochondrial } \\
\hline Nadl & $2(0.5)$ & 4 & 0.48 & 0.001 & 0.3 & 0.56 & 0 & 0 & 1.08 & 0.59 & 0.89 \\
\hline Nad5 & $2(0.8)$ & 3 & 0.34 & 0.001 & 0.3 & 0.34 & 0 & 0 & 0.19 & 0.59 & 0.55 \\
\hline \multicolumn{12}{|l|}{ Nuclear } \\
\hline$\beta$-tubulin & $5(1.4)$ & 9 & 0.34 & 0.001 & 0.68 & 0.5 & 0.40 & 1 & -0.42 & 0.87 & 0.51 \\
\hline gpi & $2(0.5)$ & 3 & 0.04 & 0 & 0.27 & 0.04 & 0 & 0 & -0.95 & 0.56 & 0.07 \\
\hline nrITS & $13(2.1)$ & 19 & 0.31 & 0.001 & 1.75 & 0.57 & 0.001 & 3 & -1.45 & 0.59 & -0.25 \\
\hline nrLSU & $19(2.7)$ & 41 & 0.19 & 0.001 & 2.56 & 0.83 & 0.001 & 8 & -1.57 & 0.1 & -0.08 \\
\hline TigA & $7(1.8)$ & 15 & 0.60 & 0.004 & 0.94 & 0.8 & 4.2 & 2 & -0.28 & 1.02 & 0.67 \\
\hline
\end{tabular}

a Abbreviations: s, number of polymorphisms; h, number of haplotypes per gene; Hd, haplotype diversity; $\pi$, nucleotide diversity; $\theta \mathrm{w}$, Watterson's theta estimator per gene from sequence; $\mathrm{k}$, average number of nucleotide differences.

${ }^{\mathrm{b}} \mathrm{R}$, recombination parameter and $\mathrm{RM}$, minimum number of recombination events.

${ }^{\mathrm{c}}$ For Fu and Li's D and Fu and Li's F, none of the neutrality tests were significant at 0.05 .

${ }^{\mathrm{d}}$ Subunit $1(\mathrm{Nadl})$ and 5 (Nad5) of the NADH dehydrogenase gene, $\beta$-tubulin, glucose phosphate isomerase (gpi), nuclear ribosomal internal transcribed spacer (nrITS), large nuclear ribosomal subunit (nrLSU), and TigA gene fusion protein (TigA). 

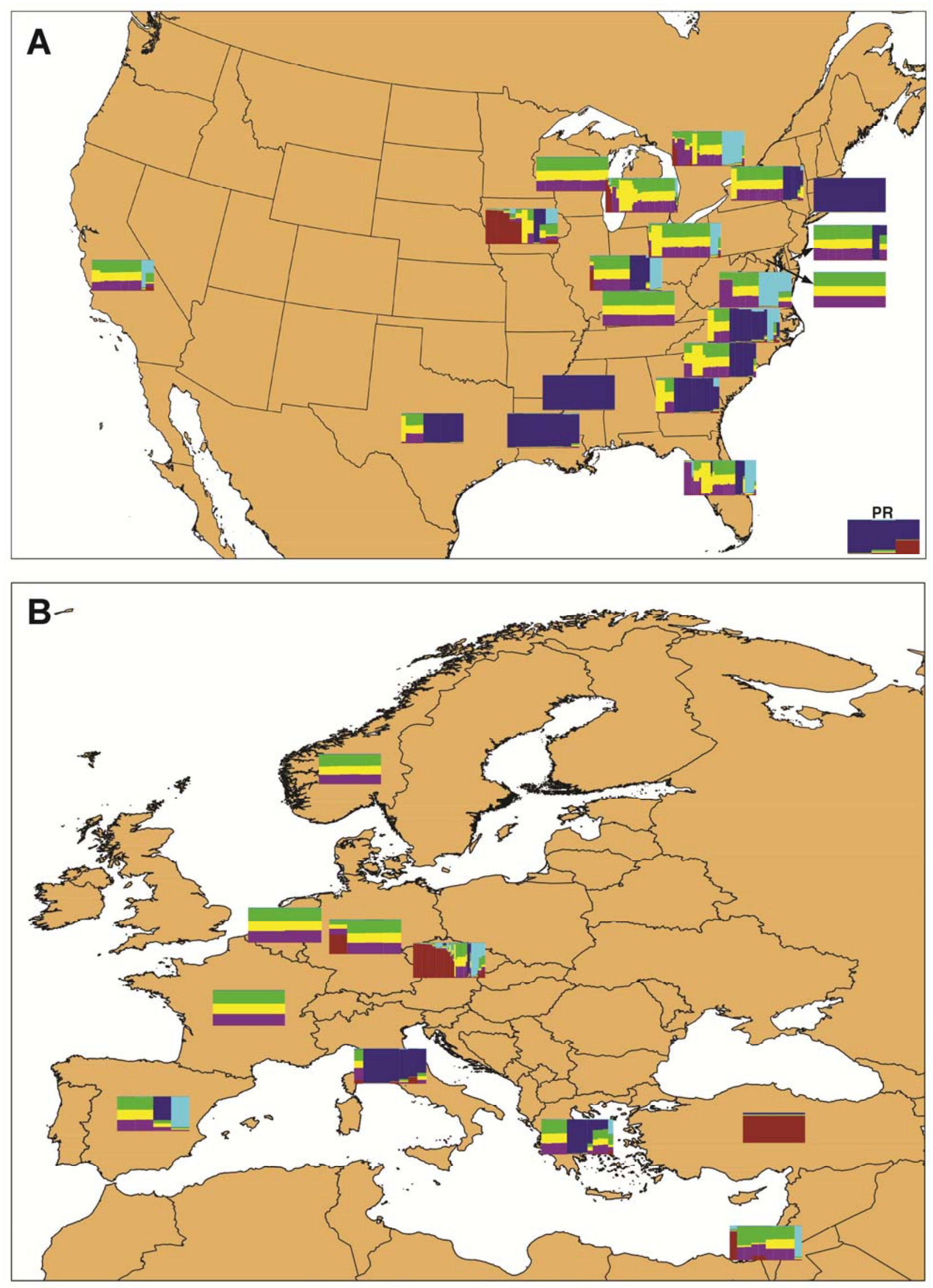

Fig. 1. Genetic structure of Pseudoperonospora cubensis isolates by geographic origin. Geographic origin: A, North America; B, Europe and Asia. Each isolate is represented by a thin bar, which is partitioned into colored segments each representing the individual's proportionate genetic membership in a given Kth cluster. Cluster colors correspond to red $=1$, purple $=2$, yellow $=3$, green $=4$, blue $=5$, and turquoise $=6$. 
quently sent to the Michigan State University Research Technology Support Facility (East Lansing) for sequencing. An ABI PRISM 3100 Genetic Analyzer (Applied Biosystems) was used to complete two cycle-sequencing reactions $(1 \mu \mathrm{l}$ of purified PCR product, $3 \mu \mathrm{l}$ of primer, and $8 \mu \mathrm{l}$ of sterile water) directly from the clean PCR products. Haplotypes were resolved according to the methods outlined by Quesada-Ocampo et al. (44).

Sequence analysis. Lasergene SeqMan Pro (version 8.0; DNASTAR Inc.) was used for manual editing of base calls and for sequence alignment. FASTA files were imported into MacClade (31) and converted to NEXUS files for subsequent analyses. Sequences from mitochondrial and nuclear loci were analyzed individually and collapsed into unique single-locus haplotypes using DnaSPv5 (30). Haplotypes found for each gene were deposited in GenBank under accession numbers HQ636479 to HQ636573. Haplotype sequences were compared with sequence data from the $P$. cubensis genome project (courtesy B. Day, MSU) by using nucleotide BLAST $(1,2)$ to determine that data corresponded to the $P$. cubensis target genes.

DNA sequence variability, neutrality tests, and recombination. Polymorphism, neutrality, and recombination analysis were carried out on individual loci for all samples and for isolates grouped in host (Cucumis sativus or other host), geographic (continent, country, United States or other, and U.S. state), and temporal (U.S. isolates only: 1982, 2004, 2007, 2008, and 2009) categories using the program DnaSPv5. Only categories with at least eight individuals were included for these analyses following the methods of previous studies $(18,44)$. Using DnaSPv5, sequence diversity estimates and statistics, including the number of segregating sites (s) and haplotypes (h), haplotype diversity (37), Tajima's $\pi$ (59), the average number of pairwise nucleotide differences $(\mathrm{k})(59)$, and Watterson's theta $(\theta \mathrm{w})$ per sequence (66) were calculated. Fu and Li's D and F, and Tajima's D were used to assess whether sequence variation in each gene for all isolates deviated from neutrality $(15,60)$; and the recombination parameter (R) per gene (22) and minimum number of recombination events (RM) (23) were also estimated for each gene and all isolates.

Population subdivision analysis. The model-based Bayesian clustering algorithm implemented in Structure $2.3 \mathrm{X}$ was used to assess population subdivision (43). Following preliminary analyses, the values for burnin, chain replication, and lambda were chosen to be $300,000,100,000$, and 0.8 , respectively. The optimal number of populations $(K)$ was determined by comparing posterior
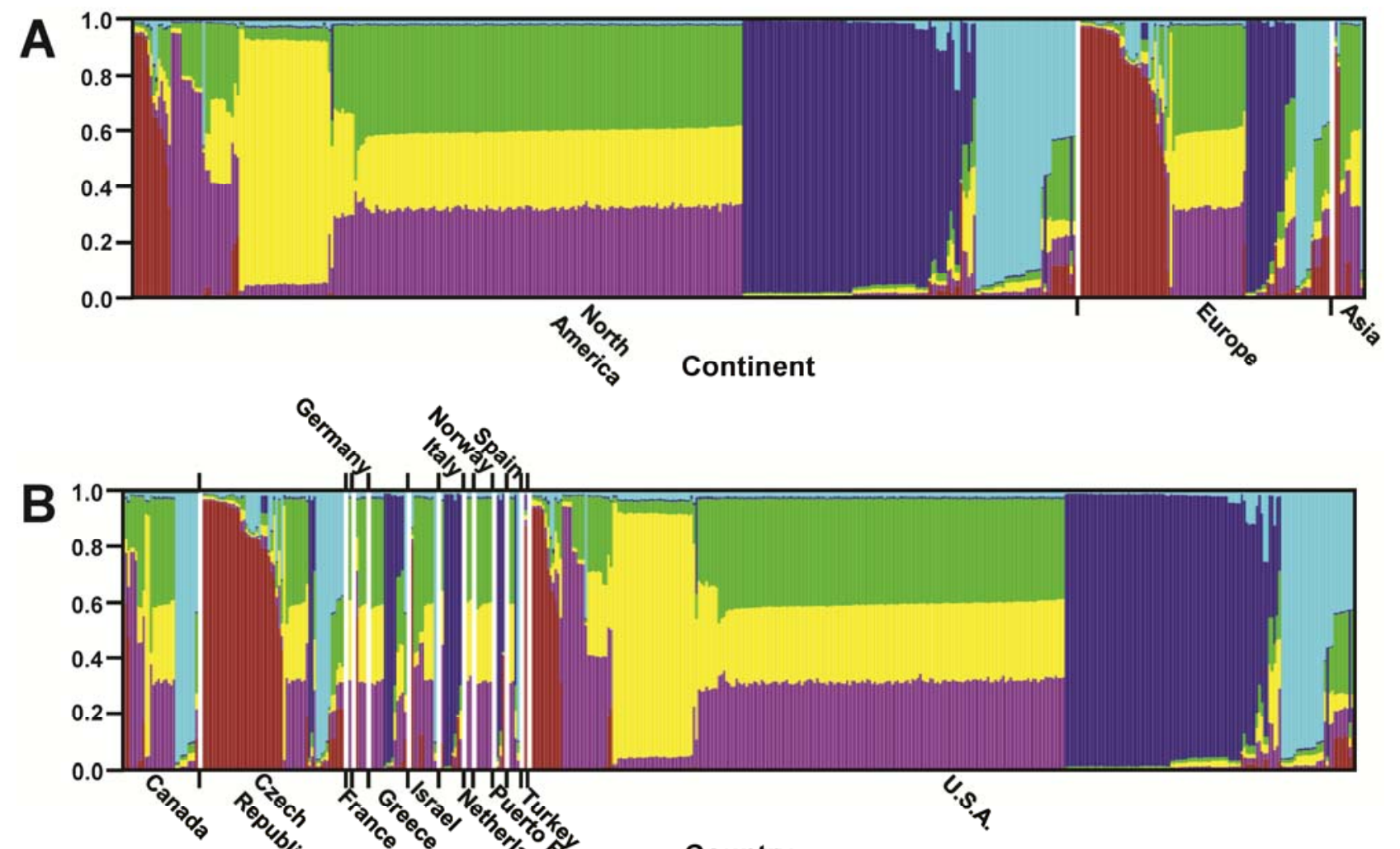

Country

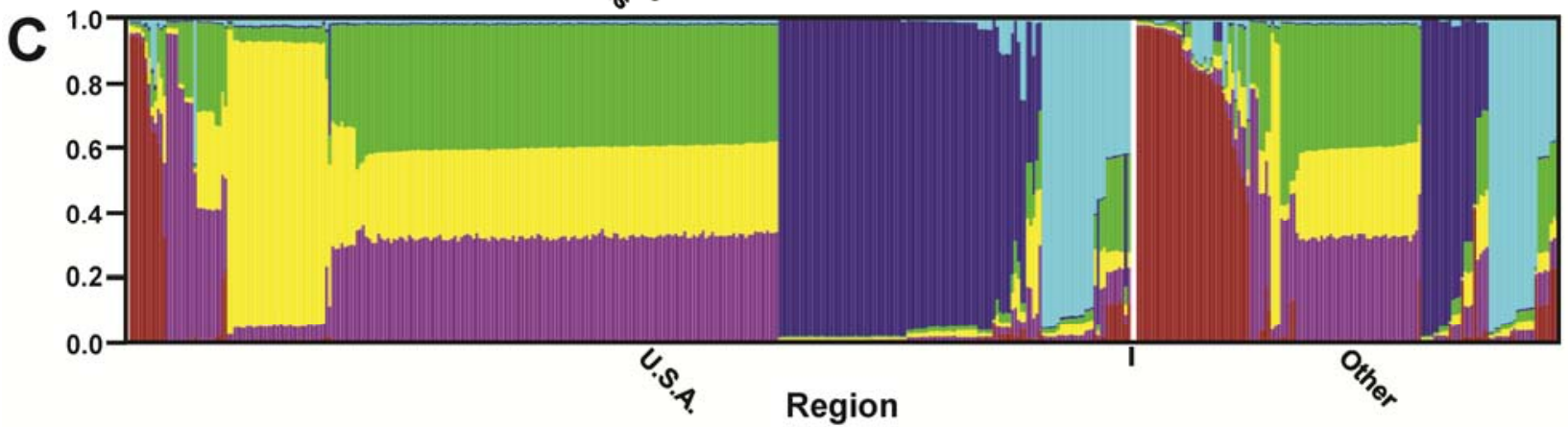

Fig. 2. Genetic structure of Pseudoperonospora cubensis isolates grouped by geographic categories: $\mathbf{A}$, continent; $\mathbf{B}$, country; and $\mathbf{C}$, region. Geographic origin abbreviations: U.S.A. = United States of America, CA = California, DE = Delaware, FL = Florida, GA = Georgia, IA = Iowa, IN = Indiana, LA = Louisiana, MI = Michigan, NC = North Carolina, NY = New York, $\mathrm{OH}=$ Ohio, $\mathrm{SC}=$ South Carolina, $\mathrm{TX}=$ Texas, VA = Virginia, and WI = Wisconsin.. Each isolate is represented by a thin bar, which is partitioned into colored segments each representing the individual's proportionate genetic membership in a given Kth cluster. Cluster colors correspond to red $=1$, purple $=2$, yellow $=3$, green $=4$, blue $=5$, and turquoise $=6$. A white space separates individual subcategories . 
distribution likelihoods among three independent runs of $K=1$ to $K=40$ using the established parameters. Data included all loci individually coded by haplotype and were analyzed under the admixture model with correlated allele frequencies and without previous population information. To visualize the distribution of clusters in predefined categories, population structure figures with prior population information, obtained from the defined geographic, host, and year categories and sorted by the proportional membership (Q) of an isolate in a cluster, were generated using the Population Sorting Tool, a graphic editing program created in R (45) (J. J. Morrice, unpublished data). Genetic differentiation indexes (Wright's $\mathrm{F}_{\mathrm{ST}}$ ) were calculated for isolates grouped by geographic region, host, and year categories using DnaSPv5. Genetic differentiation was interpreted to be low, moderate, or high according to the standards in Hartl and Clark (19). Statistical significance was determined for each index by running 5,000 permutations $(\alpha=0.05)$.

\section{Results}

In total, 465 P. cubensis isolates originating from diverse hosts (C. sativus, C. melo, Cucurbita pepo, C. maxima, and C. moschata) and geographic regions (three continents, 13 countries, and 19 U.S. states) were genotyped for two mitochondrial and five nuclear regions (Table 1). All genes were polymorphic but nuclear genes were more variable than mitochondrial genes, as indicated by diversity estimates (Table 2). In all, 50 polymorphisms were detected, and nuclear genes presented low values of R and RM. Fu and Li's D and F and Tajima's D indicated no significant deviation in neutrality for any of the genes. No unique haplotypes were found in the mitochondrial genes. Unique single-locus haplotypes occurred in all nuclear genes except gpi, and their frequency ranged between 13\% (TigA) and 40\% (nrLSU) (data not shown). In total, there were 322 multilocus genotypes within the sample of 465 isolates. Isolates that shared a multilocus genotype were found in all three continents, across multiple hosts, and across multiple years, suggesting that some genotypes are widespread and persistent.

Bayesian clustering revealed significant population structure by geographic region (Figs. 1 and 2) and host (Fig. 3), where some clusters occurred more frequently in some categories than others. However, the analysis showed no direct correspondence between

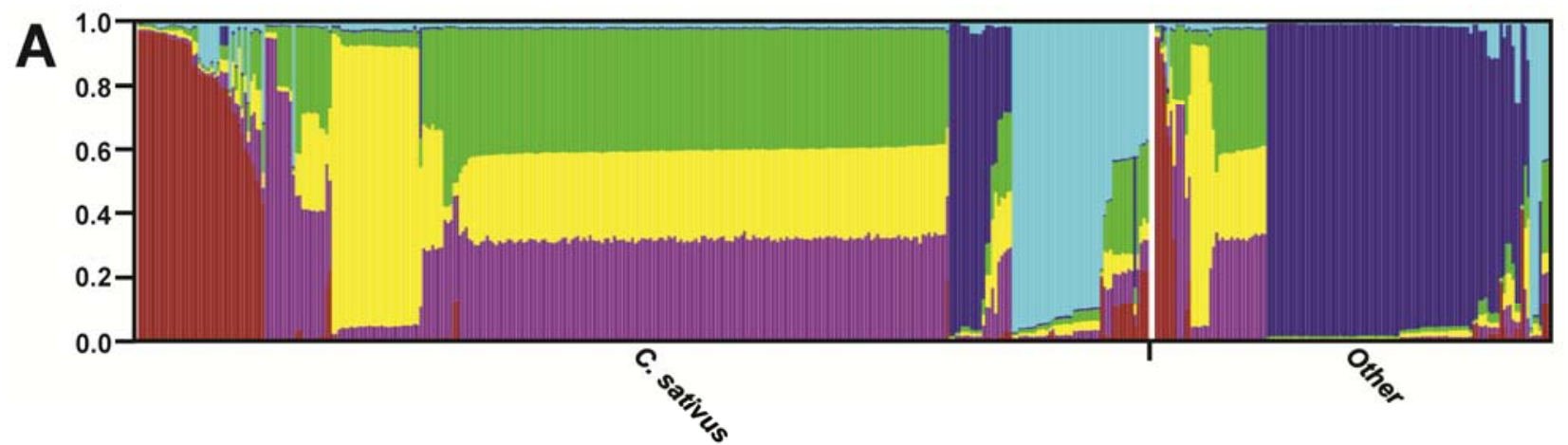

Host species

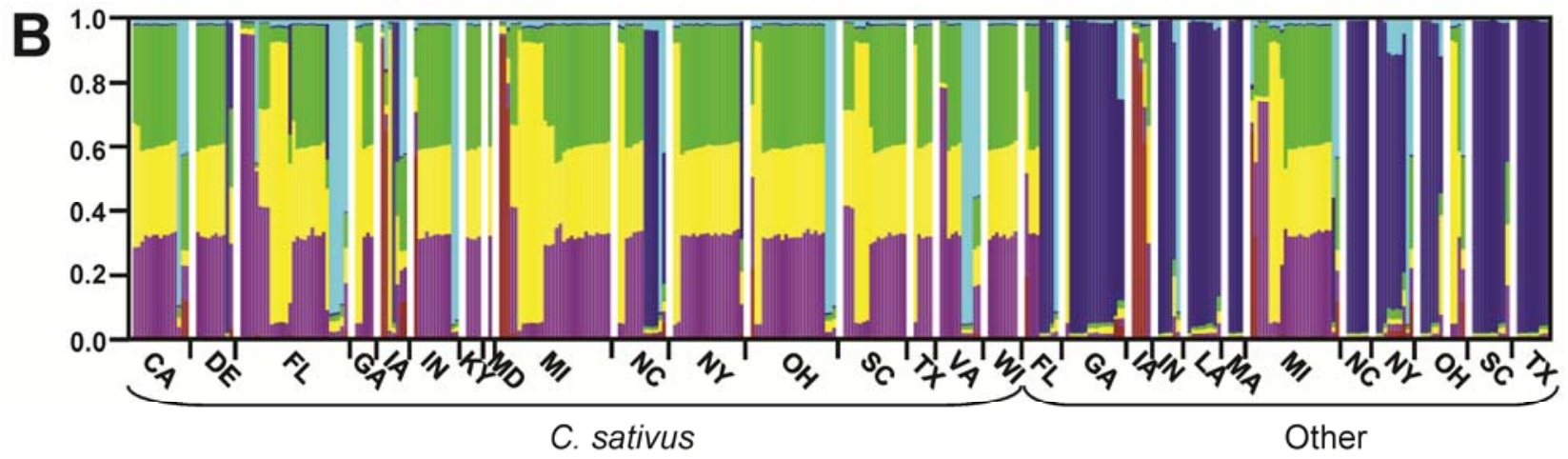

Host species (U.S.A. state)

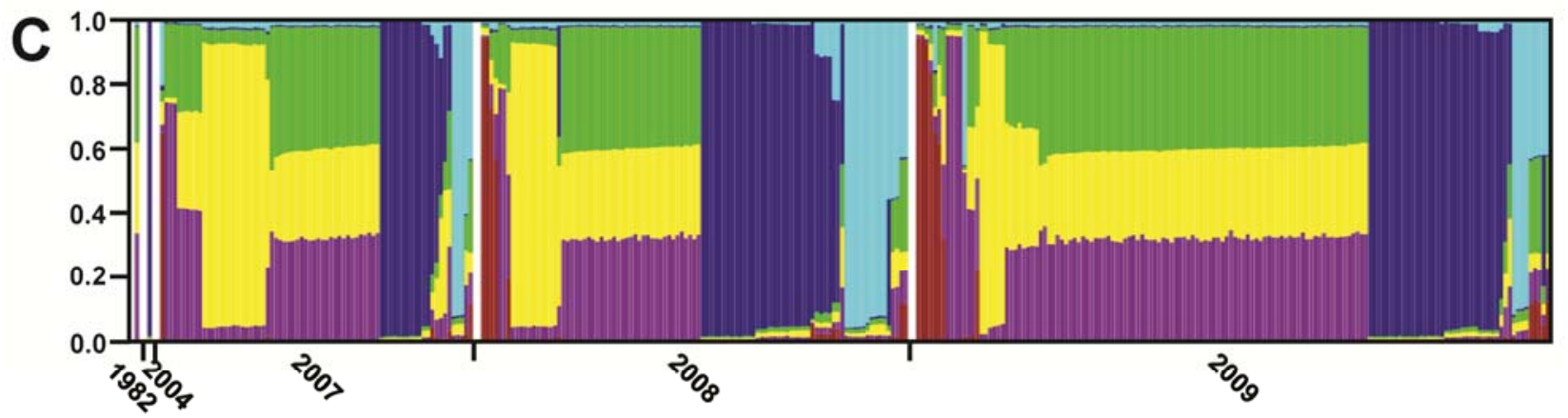

Year (U.S.A. isolates only)

Fig. 3. Genetic structure of Pseudoperonospora cubensis isolates grouped by predefined host and year categories: A, Cucumis sativus or other; B, hosts (U.S. state); and C, year. Each isolate is represented by a thin bar, which is partitioned into colored segments each representing the individual's proportionate genetic membership in a given Kth cluster. Cluster colors correspond to red $=1$, purple $=2$, yellow $=3$, green $=4$, blue $=5$, and turquoise $=6$. A white space separates individual subcategories. 
groupings of $P$. cubensis isolates in the predefined host and geography categories and inferred genetic clusters. The highest likelihood scores were observed when the number of clusters was set to six ( $K$ $=6, \ln \mathrm{P}=-2,877 ; K=5, \ln \mathrm{P}=-2,987$; and $K=7, \ln \mathrm{P}=-2,940)$, and individual ancestry coefficients were highly consistent across replicate runs. The bar plots indicated that approximately half of the isolates were assigned predominantly to clusters $1,2,3,4,5$, or 6 and the other half were assigned partial membership in clusters 2 , 3 , and 4 (referred to as mixed isolates in this article, based on genetic clustering).

P. cubensis population structure by geography. Population structure was detected when data were analyzed using geographic categories as prior population information (Figs. 1 and 2). $\mathrm{F}_{\mathrm{ST}}$ values indicated low differentiation between U.S. and non-U.S. isolates (Table 3), and diversity estimates were comparable between isolates from both categories (Table 4). Isolates belonging to each of the clusters were present in the sampling from North America and Europe (Figs. 1 and 2A). Isolates belonging predominantly to cluster 1 occurred more frequently in Europe (69\% of cluster 1 isolates), whereas clusters 2,3 , and 5 were more frequently found in North America $(100,97$, and $85 \%$ of isolates in each cluster, respectively). $\mathrm{F}_{\mathrm{ST}}$ estimates indicated moderate differentiation between isolates from Western Asia and isolates from Europe and North America (Table 3). Diversity estimates were higher for iso- lates from Europe and North America than those obtained from Western Asia (Table 4).

When isolates were grouped by country, mixed isolates were present in the samples collected from all countries except Turkey (Figs. 1 and 2 B). Isolates from cluster 1 were more frequently sampled in the Czech Republic, while isolates from clusters 2, 3, and 5 were mostly found in the U.S.. Isolates from cluster 6 were mainly sampled in Canada, the Czech Republic, and the U.S.. Italy only presented isolates from cluster 5 , except for one mixed isolate (Figs. 1 and 2B). Countries (France, Germany, the Netherlands, and Norway) in Western Europe showed similar cluster composition, as did Greece and Italy. Spain showed similar cluster composition to countries in Western Europe, Greece, and Italy. Turkey and the Czech Republic displayed similar cluster composition. Israel showed similar cluster composition to the Czech Republic but not to Greece or Italy (Figs. 1 and 2B), and $\mathrm{F}_{\mathrm{ST}}$ values detected low genetic differentiation between Israel and the Czech Republic but high differentiation among Israel, Greece, and Italy. $\mathrm{F}_{\mathrm{ST}}$ values indicated high genetic differentiation between isolates from Italy and other countries (Table 3 ). $\mathrm{F}_{\mathrm{ST}}$ values established high genetic differentiation between isolates from Greece and Italy but lower than differentiation between the Czech Republic and Greece or Italy (Table 3). Differentiation estimates also indicated high differentiation between isolates from Greece and

Table 3. Genetic differentiation estimates of mitochondrial and nuclear genes for Pseudoperonospora cubensis isolates grouped by continent, country, and country group

\begin{tabular}{|c|c|c|c|c|c|c|c|c|c|c|c|c|c|c|c|c|}
\hline \multirow[b]{4}{*}{ Category $^{a}$} & \multicolumn{16}{|c|}{$\mathbf{F}_{\mathbf{S T}}^{\mathbf{b}}$} \\
\hline & \multicolumn{4}{|c|}{ Continent } & \multicolumn{10}{|c|}{ Country } & \multirow{2}{*}{\multicolumn{2}{|c|}{$\begin{array}{c}\begin{array}{c}\text { Country } \\
\text { group }\end{array} \\
\text { United States }\end{array}$}} \\
\hline & \multicolumn{2}{|c|}{ Asia } & \multicolumn{2}{|c|}{ Europe } & \multicolumn{2}{|c|}{ Canada } & \multicolumn{2}{|c|}{ Czech Rep. } & \multicolumn{2}{|c|}{ Greece } & \multicolumn{2}{|c|}{ Israel } & \multicolumn{2}{|c|}{ Italy } & & \\
\hline & M & $\mathbf{N}$ & M & $\mathbf{N}$ & $\mathbf{M}$ & $\mathbf{N}$ & M & $\mathbf{N}$ & $\mathbf{M}$ & $\mathbf{N}$ & $\mathbf{M}$ & $\mathbf{N}$ & $\mathbf{M}$ & $\mathbf{N}$ & M & $\mathbf{N}$ \\
\hline \multicolumn{17}{|l|}{ Continent } \\
\hline Europe & $0.08^{*}$ & $0.05^{*}$ & $\ldots$ & $\ldots$ & $\ldots$ & $\ldots$ & $\ldots$ & $\ldots$ & $\ldots$ & $\ldots$ & $\ldots$ & $\ldots$ & $\ldots$ & $\ldots$ & $\ldots$ & $\ldots$ \\
\hline North America & $0.16^{*}$ & $0.05^{*}$ & 0.01 & $0.06^{*}$ & $\ldots$ & $\ldots$ & $\ldots$ & $\ldots$ & $\ldots$ & $\ldots$ & $\ldots$ & $\ldots$ & $\ldots$ & $\ldots$ & $\ldots$ & $\ldots$ \\
\hline \multicolumn{17}{|l|}{ Country } \\
\hline Czech Republic & $\ldots$ & $\ldots$ & $\ldots$ & $\ldots$ & 0.01 & $0.12 *$ & & $\ldots$ & $\ldots$ & $\ldots$ & $\ldots$ & $\ldots$ & $\ldots$ & $\ldots$ & $\ldots$ & $\ldots$ \\
\hline Greece & $\ldots$ & $\ldots$ & $\ldots$ & $\ldots$ & $0.27 *$ & $0.12 *$ & $0.22 *$ & $0.2 *$ & $\ldots$ & $\ldots$ & $\ldots$ & $\ldots$ & $\ldots$ & $\ldots$ & $\ldots$ & $\ldots$ \\
\hline Israel & $\ldots$ & $\ldots$ & $\ldots$ & $\ldots$ & 0 & $0.05^{*}$ & 0.01 & $0.1^{*}$ & $0.24 *$ & $0.16^{*}$ & $\ldots$ & $\ldots$ & $\ldots$ & $\ldots$ & $\ldots$ & $\ldots$ \\
\hline Italy & $\ldots$ & $\ldots$ & $\ldots$ & $\ldots$ & $0.72 *$ & $0.18 *$ & $0.68 *$ & $0.21 *$ & $0.34 *$ & $0.08 *$ & $0.72 *$ & $0.22 *$ & $\ldots$ & $\ldots$ & $\ldots$ & $\ldots$ \\
\hline United States & $\ldots$ & $\ldots$ & $\ldots$ & $\ldots$ & $0.19^{*}$ & $0.04 *$ & $0.14 *$ & $0.11^{*}$ & 0 & $0.06^{*}$ & $0.17 *$ & $0.07 *$ & $0.43^{*}$ & $0.13^{*}$ & $\ldots$ & $\ldots$ \\
\hline \multicolumn{17}{|l|}{ Country group } \\
\hline Other & $\ldots$ & $\ldots$ & $\ldots$ & $\ldots$ & $\ldots$ & $\ldots$ & $\ldots$ & $\ldots$ & $\ldots$ & $\ldots$ & $\ldots$ & $\ldots$ & $\ldots$ & $\ldots$ & $0.04 *$ & $0.03^{*}$ \\
\hline
\end{tabular}

${ }^{a}$ Average values for mitochondrial $(\mathrm{M})$ and nuclear genes $(\mathrm{N})$ are presented; $*=$ significant at 0.05 .

${ }^{\mathrm{b}}$ Categories with less than eight isolates were excluded from analyses and are not shown.

Table 4. Diversity estimates of mitochondrial and nuclear genes for Pseudoperonospora cubensis isolates grouped by continent, country, and country group

\begin{tabular}{|c|c|c|c|c|c|c|c|c|c|}
\hline \multirow[b]{3}{*}{ Category $^{b}$} & \multirow[b]{3}{*}{ Isolates $^{\mathrm{c}}$} & \multicolumn{8}{|c|}{ Diversity estimates ${ }^{a}$} \\
\hline & & \multicolumn{2}{|c|}{ Hd } & \multicolumn{2}{|c|}{$\pi$} & \multicolumn{2}{|c|}{$\theta \mathbf{w}$} & \multicolumn{2}{|c|}{$\mathbf{k}$} \\
\hline & & $\mathbf{M}$ & $\mathbf{N}$ & $\mathbf{M}$ & $\mathbf{N}$ & $\mathbf{M}$ & $\mathbf{N}$ & $\mathbf{M}$ & $\mathbf{N}$ \\
\hline \multicolumn{10}{|l|}{ Continent } \\
\hline Asia & 11 & 0.16 & 0.32 & 0.001 & 0.001 & 0.17 & 1.26 & 0.16 & 0.84 \\
\hline Europe & 95 & 0.37 & 0.44 & 0.002 & 0.001 & 0.29 & 1.20 & 0.40 & 1.14 \\
\hline North America & 359 & 0.43 & 0.25 & 0.001 & 0.001 & 0.31 & 1.09 & 0.47 & 0.35 \\
\hline \multicolumn{10}{|l|}{ Country } \\
\hline Canada & 29 & 0.21 & 0.30 & 0.002 & 0.001 & 0.13 & 0.52 & 0.21 & 0.40 \\
\hline Czech Republic & 56 & 0.27 & 0.48 & 0 & 0.001 & 0.33 & 1.21 & 0.28 & 1.46 \\
\hline Greece & 14 & 0.47 & 0.31 & 0 & 0.001 & 0.31 & 0.26 & 0.47 & 0.35 \\
\hline Israel & 10 & 0.18 & 0.25 & 0.002 & 0.001 & 0.18 & 0.68 & 0.18 & 0.54 \\
\hline Italy & 8 & 0.34 & 0.26 & 0.001 & 0.001 & 0.39 & 0.96 & 0.34 & 0.72 \\
\hline United States & 327 & 0.43 & 0.24 & 0 & 0.001 & 0.31 & 0.82 & 0.47 & 0.33 \\
\hline \multicolumn{10}{|l|}{ Country group } \\
\hline United States & 327 & 0.43 & 0.24 & 0.001 & 0.001 & 0.31 & 0.82 & 0.47 & 0.33 \\
\hline Other & 138 & 0.36 & 0.40 & 0.001 & 0.002 & 0.27 & 1.29 & 0.39 & 1.01 \\
\hline
\end{tabular}

${ }^{a}$ Average values for mitochondrial $(\mathrm{M})$ and nuclear $(\mathrm{N})$ genes are presented. Hd, haplotype diversity; $\pi$, nucleotide diversity; $\theta \mathrm{w}$, Watterson's theta estimator per gene from sequence; $\mathrm{k}$, average number of nucleotide differences.

${ }^{\mathrm{b}}$ Categories with less than eight isolates were excluded from analyses and are not shown.

c Number of isolates. 
from other countries, except for the U.S.. Moderate to low differentiation was detected among isolates from the Czech Republic, Israel, Canada, and the U.S.. Diversity estimates were higher for isolates from the Czech Republic, Greece, and Italy, while isolates from Canada, Israel, and the U.S. presented lower values (Table 4).

When isolates were grouped by U.S. state (Figs. 1A and 3B), it was observed that the pattern of cluster occurrence was similar in Georgia, New York, South Carolina, and Texas; North Carolina, Indiana, and Florida; California, Delaware, Kentucky, Maryland, Michigan, Ohio, Virginia, and Wisconsin; and Louisiana, Massachusetts, and Mississippi. A greater number of clusters were detected in the sample from Iowa, and the pattern of cluster occur- rence was unique (Figs. 1A and $3 \mathrm{~B}$ ). However, $\mathrm{F}_{\mathrm{ST}}$ values indicated low differentiation between isolates from Michigan, Ohio, Wisconsin, Iowa, and California. Similarly, low differentiation was observed between isolates from North Carolina, South Carolina, Georgia, Indiana, and Texas. Isolates from New York, Delaware, Virginia, and Florida also showed low differentiation among each other. Isolate composition from Louisiana had very high differentiation compared with all other states (Table 5) and these isolates also presented the lowest diversity estimates of any state (Table 6). Diversity estimates were high for isolates from Georgia, Iowa, and North Carolina, followed by moderate to high estimates obtained for isolates from Indiana (Table 6). Isolates from Delaware, Michigan, New York, Ohio, South Carolina, Virginia, and Wisconsin

Table 5. Genetic differentiation estimates for Pseudoperonospora cubensis isolates grouped by U.S. state ${ }^{\mathrm{a}}$

\begin{tabular}{|c|c|c|c|c|c|c|c|c|c|c|c|c|c|c|}
\hline \multirow[b]{3}{*}{ State $^{c}$} & \multicolumn{14}{|c|}{$\mathbf{F}_{\mathrm{ST}}^{\mathbf{b}}$} \\
\hline & \multicolumn{2}{|c|}{ CA } & \multicolumn{2}{|c|}{ DE } & \multicolumn{2}{|c|}{ FL } & \multicolumn{2}{|c|}{ GA } & \multicolumn{2}{|c|}{ IA } & \multicolumn{2}{|c|}{ IN } & \multicolumn{2}{|c|}{ LA } \\
\hline & $\mathbf{M}$ & $\mathbf{N}$ & M & $\mathbf{N}$ & $\mathbf{M}$ & $\mathbf{N}$ & $\mathbf{M}$ & $\mathbf{N}$ & $\mathbf{M}$ & $\mathbf{N}$ & $\mathbf{M}$ & $\mathbf{N}$ & $\mathbf{M}$ & $\mathbf{N}$ \\
\hline $\mathrm{DE}$ & 0 & $0.05^{*}$ & & & $\ldots$ & $\ldots$ & $\ldots$ & $\ldots$ & $\ldots$ & $\ldots$ & $\ldots$ & $\ldots$ & $\ldots$ & $\ldots$ \\
\hline FL & $0.07 *$ & $0.04^{*}$ & 0.02 & 0.02 & $\ldots$ & $\ldots$ & $\ldots$ & $\ldots$ & $\ldots$ & $\ldots$ & $\ldots$ & $\ldots$ & $\ldots$ & $\ldots$ \\
\hline GA & $0.56^{*}$ & $0.09 *$ & $0.43^{*}$ & $0.05 *$ & $0.36^{*}$ & $0.04 *$ & $\ldots$ & $\ldots$ & $\ldots$ & $\ldots$ & $\ldots$ & $\ldots$ & $\ldots$ & $\ldots$ \\
\hline IA & 0.03 & $0.09^{*}$ & 0 & $0.15^{*}$ & 0.03 & $0.13^{*}$ & $0.37 *$ & $0.16^{*}$ & $\ldots$ & $\ldots$ & $\ldots$ & $\ldots$ & $\ldots$ & $\ldots$ \\
\hline IN & $0.15^{*}$ & $0.04 *$ & 0.03 & 0.01 & 0 & 0.02 & $0.2 *$ & 0.006 & 0.04 & $0.13 *$ & $\ldots$ & 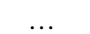 & $\ldots$ & $\ldots$ \\
\hline LA & $0.97 *$ & $0.09 *$ & $0.89^{*}$ & $0.11^{*}$ & $0.81^{*}$ & $0.07 *$ & $0.29 *$ & $0.07 *$ & $0.85^{*}$ & $0.06^{*}$ & $0.68 *$ & $0.06^{*}$ & & \\
\hline MI & 0 & 0.02 & 0 & $0.05^{*}$ & $0.08^{*}$ & $0.04 *$ & $0.55^{*}$ & $0.09 *$ & 0.01 & $0.1 *$ & $0.14^{*}$ & $0.06^{*}$ & $0.97 *$ & $0.08 *$ \\
\hline $\mathrm{NC}$ & $0.42 *$ & $0.06^{*}$ & $0.28 *$ & $0.08 *$ & $0.21 *$ & $0.08^{*}$ & 0 & $0.08 *$ & $0.23^{*}$ & $0.05^{*}$ & 0.07 & $0.05^{*}$ & $0.42 *$ & $0.06^{*}$ \\
\hline NY & $0.18^{*}$ & $0.04 *$ & 0.01 & 0 & 0.04 & 0.01 & $0.23^{*}$ & $0.04 *$ & 0.02 & $0.14 *$ & 0 & 0.01 & $0.75^{*}$ & $0.09 *$ \\
\hline $\mathrm{OH}$ & 0 & 0 & 0.02 & $0.03 *$ & $0.06 *$ & $0.03 *$ & $0.5^{*}$ & 0.02 & 0.05 & $0.11 *$ & $0.14 *$ & $0.04 *$ & $0.95^{*}$ & $0.09 *$ \\
\hline $\mathrm{SC}$ & $0.29 *$ & $0.08^{*}$ & $0.13^{*}$ & 0.01 & $0.1^{*}$ & 0.01 & $0.11^{*}$ & 0.03 & $0.09^{*}$ & $0.16^{*}$ & 0 & 0.01 & $0.58^{*}$ & $0.1^{*}$ \\
\hline TX & $0.56^{*}$ & $0.11^{*}$ & $0.41 *$ & $0.05^{*}$ & $0.36^{*}$ & $0.05 *$ & 0 & 0.02 & $0.35^{*}$ & $0.18^{*}$ & $0.19 *$ & 0.02 & $0.28 *$ & $0.1 *$ \\
\hline VA & 0.03 & $0.12 *$ & $0.07 *$ & $0.1^{*}$ & $0.05^{*}$ & $0.15^{*}$ & $0.51^{*}$ & $0.12 *$ & $0.1^{*}$ & $0.23^{*}$ & $0.13^{*}$ & $0.09^{*}$ & $0.91 *$ & $0.21 *$ \\
\hline \multirow[t]{3}{*}{ WI } & 0 & $0.02^{*}$ & 0 & $0.06^{*}$ & $0.12 *$ & 0.03 & $0.59 *$ & $0.1^{*}$ & 0.03 & $0.12 *$ & $0.18 *$ & $0.05 *$ & $1 *$ & $0.12 *$ \\
\hline & \multicolumn{2}{|c|}{ MI } & \multicolumn{2}{|c|}{ NC } & \multicolumn{2}{|c|}{ NY } & \multicolumn{2}{|c|}{$\mathrm{OH}$} & \multicolumn{2}{|c|}{$\mathrm{SC}$} & \multicolumn{2}{|c|}{ TX } & \multicolumn{2}{|c|}{ VA } \\
\hline & $\mathbf{M}$ & $\mathbf{N}$ & $\mathbf{M}$ & $\mathbf{N}$ & $\mathbf{M}$ & $\mathbf{N}$ & $\mathbf{M}$ & $\mathbf{N}$ & $\mathbf{M}$ & $\mathbf{N}$ & $\mathbf{M}$ & $\mathbf{N}$ & $\mathbf{M}$ & $\mathbf{N}$ \\
\hline $\mathrm{NC}$ & $0.4 *$ & $0.09^{*}$ & & & $\ldots$ & $\ldots$ & $\ldots$ & $\ldots$ & $\ldots$ & $\ldots$ & $\ldots$ & $\ldots$ & $\ldots$ & $\ldots$ \\
\hline NY & $0.17 *$ & $0.04 *$ & $0.13 *$ & $0.08^{*}$ & $\ldots$ & $\ldots$ & $\ldots$ & $\ldots$ & $\ldots$ & $\ldots$ & $\ldots$ & $\ldots$ & $\ldots$ & $\ldots$ \\
\hline $\mathrm{OH}$ & 0.02 & 0.01 & $0.4^{*}$ & $0.09 *$ & $0.19^{*}$ & $0.02 *$ & $\ldots$ & $\ldots$ & $\ldots$ & $\ldots$ & $\ldots$ & $\ldots$ & $\ldots$ & $\ldots$ \\
\hline $\mathrm{SC}$ & $0.28^{*}$ & $0.07 *$ & 0.02 & $0.09 *$ & 0 & 0.01 & $0.19 *$ & $0.02 *$ & $\ldots$ & $\ldots$ & $\ldots$ & $\ldots$ & $\ldots$ & $\ldots$ \\
\hline TX & $0.55^{*}$ & $0.1^{*}$ & 0 & $0.11^{*}$ & $0.22 *$ & $0.03 *$ & 0 & 0.01 & $0.2^{*}$ & $0.02 *$ & . & & $\ldots$ & $\ldots$ \\
\hline VA & $0.08^{*}$ & $0.13^{*}$ & $0.37 *$ & $0.18^{*}$ & $0.2^{*}$ & $0.09 *$ & $0.2 *$ & $0.03 *$ & $0.29 *$ & $0.06 *$ & 0 & $0.11 *$ & $\ldots$ & $\ldots$ \\
\hline WI & 0 & 0.02 & $0.4^{*}$ & $0.11^{*}$ & $0.21 *$ & $0.02 *$ & $0.2^{*}$ & $0.09 *$ & $0.55^{*}$ & $0.09 *$ & 0.04 & 0 & $0.1^{*}$ & $0.1^{*}$ \\
\hline
\end{tabular}

${ }^{\text {a }} \mathrm{CA}=$ California, $\mathrm{DE}=$ Delaware, $\mathrm{FL}=$ Florida, GA = Georgia, IA = Iowa, IN = Indiana, LA = Louisiana, MI = Michigan, NC = North Carolina, NY =

New York, $\mathrm{OH}=$ Ohio, $\mathrm{SC}=$ South Carolina, $\mathrm{TX}=$ Texas, $\mathrm{VA}=$ Virginia, and $\mathrm{WI}=$ Wisconsin .

${ }^{\mathrm{b}}$ Average values for mitochondrial $(\mathrm{M})$ and nuclear genes $(\mathrm{N})$ are presented; * $=$ significant at 0.05 .

${ }^{\mathrm{c}}$ Categories with less than eight isolates were excluded from analyses and are not shown.

Table 6. Diversity estimates of mitochondrial and nuclear genes for Pseudoperonospora cubensis isolates grouped by U.S. state

\begin{tabular}{|c|c|c|c|c|c|c|c|c|c|}
\hline \multirow[b]{3}{*}{ Category $^{\mathbf{b}}$} & \multirow[b]{3}{*}{ Isolates $^{c}$} & \multicolumn{8}{|c|}{ Diversity estimates $^{\mathbf{a}}$} \\
\hline & & \multicolumn{2}{|c|}{ Hd } & \multicolumn{2}{|c|}{$\pi$} & \multicolumn{2}{|c|}{$\theta \mathbf{w}$} & \multicolumn{2}{|c|}{$\mathbf{k}$} \\
\hline & & M & $\mathbf{N}$ & M & $\mathbf{N}$ & M & $\mathbf{N}$ & M & $\mathbf{N}$ \\
\hline California & 15 & 0.07 & 0.15 & 0 & 0.001 & 0.15 & 0.10 & 0.07 & 0.15 \\
\hline Delaware & 10 & 0.20 & 0.17 & 0.001 & 0.001 & 0.35 & 0.11 & 0.20 & 0.17 \\
\hline Florida & 38 & 0.35 & 0.24 & 0.001 & 0.001 & 0.36 & 0.45 & 0.37 & 0.32 \\
\hline Georgia & 21 & 0.58 & 0.27 & 0.002 & 0.001 & 0.42 & 0.28 & 0.65 & 0.39 \\
\hline Iowa & 12 & 0.30 & 0.34 & 0.001 & 0.001 & 0.50 & 0.48 & 0.32 & 0.45 \\
\hline Indiana & 18 & 0.49 & 0.27 & 0.002 & 0.001 & 0.44 & 0.39 & 0.54 & 0.36 \\
\hline Louisiana & 9 & 0 & 0.31 & 0 & 0.001 & 0 & 0.35 & 0 & 0.36 \\
\hline Michigan & 54 & 0.06 & 0.21 & 0 & 0.001 & 0.33 & 0.53 & 0.06 & 0.32 \\
\hline $\mathrm{NC}$ & 23 & 0.62 & 0.27 & 0.002 & 0.001 & 0.41 & 0.32 & 0.72 & 0.34 \\
\hline New York & 25 & 0.38 & 0.17 & 0.001 & 0.001 & 0.27 & 0.18 & 0.38 & 0.21 \\
\hline Ohio & 27 & 0.10 & 0.14 & 0 & 0.001 & 0.13 & 0.22 & 0.10 & 0.19 \\
\hline South Carolina & 27 & 0.51 & 0.20 & 0.002 & 0.001 & 0.39 & 0.22 & 0.56 & 0.27 \\
\hline Texas & 14 & 0.13 & 0.52 & 0 & 0.003 & 0.31 & 0.25 & 0.13 & 0.52 \\
\hline Virginia & 11 & 0.20 & 0.52 & 0.001 & 0.003 & 0.35 & 0.28 & 0.20 & 0.52 \\
\hline Wisconsin & 8 & 0.46 & 0.58 & 0.001 & 0.004 & 0.48 & 0.82 & 0.50 & 0.84 \\
\hline
\end{tabular}

${ }^{a}$ Average values for mitochondrial $(\mathrm{M})$ and nuclear $(\mathrm{N})$ genes are presented. Hd, haplotype diversity; $\pi$, nucleotide diversity; $\theta \mathrm{w}$, Watterson's theta estimator per gene from sequence; $\mathrm{k}$, average number of nucleotide differences.

${ }^{\mathrm{b}}$ Categories with less than eight isolates were excluded from analyses and are not shown.

${ }^{c}$ Number of isolates. 
presented moderate values for diversity estimates, whereas isolates from California and Texas showed moderate to low values (Table 6).

Cluster occurrence for isolates from Ontario, Canada, was similar to what was observed for isolates from California, Delaware, Kentucky, Maryland, Michigan, Ohio, Virginia, and Wisconsin (Fig. 1A). When Ontario was analyzed for genetic differentiation with individual U.S. states, $\mathrm{F}_{\mathrm{ST}}$ values indicated that isolates from Ohio had low differentiation with Ontario (Table 7). Isolates from Michigan, New York, Wisconsin, and Virginia presented low to moderate differentiation with Ontario isolates. Moderate to high differentiation was observed between Ontario isolates and isolates from other states. Diversity estimates for Ontario isolates were lower than those obtained for isolates from Georgia, Iowa, Indiana, North Carolina, and South Carolina; comparable with isolates from

Table 7. Genetic differentiation estimates of mitochondrial and nuclear genes for Pseudoperonospora cubensis isolates from Ontario, Canada with isolates from U.S. states

\begin{tabular}{lll}
\hline & \multicolumn{2}{c}{$\mathbf{F}_{\mathbf{S T}^{\mathbf{a}}}$} \\
\cline { 2 - 3 } Category & $\mathbf{M}$ & $\mathbf{N}$ \\
\hline California & $0.05^{*}$ & 0.04 \\
Delaware & $0.09^{*}$ & $0.04^{*}$ \\
Florida & $0.05^{*}$ & $0.05^{*}$ \\
Georgia & $0.5^{*}$ & $0.07^{*}$ \\
Iowa & $0.12^{*}$ & $0.1^{*}$ \\
Indiana & $0.13^{*}$ & $0.04^{*}$ \\
Louisiana & $0.9^{*}$ & $0.13^{*}$ \\
Michigan & $0.09^{*}$ & 0.04 \\
North Carolina & $0.37^{*}$ & $0.07^{*}$ \\
New York & $0.19^{*}$ & 0.04 \\
Ohio & 0.02 & 0.04 \\
South Carolina & $0.28^{*}$ & $0.07^{*}$ \\
Texas & $0.52^{*}$ & $0.09^{*}$ \\
Virginia & 0 & $0.09^{*}$ \\
Wisconsin & $0.12^{*}$ & 0.04
\end{tabular}

a Average values for mitochondrial (left column) and nuclear genes (right column) are presented; $*=$ significant at 0.05 .

${ }^{b}$ Categories with less than eight isolates were excluded from analyses and are not shown.
Florida, Delaware, and New York; and higher than isolates from California, Louisiana, Michigan, Ohio, and Texas (Table 4).

$P$. cubensis population structure by host of origin. Population structure was revealed when isolates were organized by whether they originated from cucumber or another cucurbit host (Fig. 3A) Isolates from clusters 1, 2, 3, 4, and 6, as well as mixed isolates, were mostly sampled in Cucumis sativus $(88,77,81,86,85$, and $91 \%$ of isolates in those clusters, respectively, were sampled from C. sativus), whereas $85 \%$ of cluster 5 isolates were sampled from other hosts (Table 8; Fig. 3A). $\mathrm{F}_{\mathrm{ST}}$ values showed moderate to high differentiation between isolates from $C$. sativus and other hosts (Table 9), and diversity estimates were higher for isolates from non-cucumber hosts (Table 10).

When we further subdivided samples from each state by host $(C$. sativus or other), isolates from $C$. sativus were mostly mixed isolates, regardless of the state of origin (Fig. 3B). Most of the isolates retrieved from non-cucumber hosts belonged to cluster 5, except for isolates sampled from Iowa, Michigan, and Ohio. Most of the non-cucumber samples from Iowa belonged to cluster 1, samples from Michigan were mostly of the mixed type, and samples from Ohio showed a diversity of cluster occurrence. Mixed isolates were also found in non-cucumber isolates from Florida (Fig. 3B).

$\mathrm{F}_{\mathrm{ST}}$ values showed little to moderate differentiation between isolates from cucumber from Michigan and isolates from cucumber from other states (Table 11). Isolates from Florida cucumber showed low to moderate differentiation with isolates retrieved from cucumber in Indiana, New York, South Carolina, Virginia, and Wisconsin; moderate differentiation with Michigan cucumber isolates; and moderate to great differentiation with cucumber isolates from North Carolina and Ohio (Table 11). Moderate to high differentiation was observed for isolates retrieved from cucumber in California, Delaware, Florida, Indiana, Michigan, North Carolina, New York, Ohio, South Carolina, Virginia, and Wisconsin; and isolates retrieved from non-cucumber hosts in Florida, Georgia, Louisiana, North Carolina, South Carolina, and Texas. Noncucumber isolates retrieved from Michigan showed low to moderate differentiation with cucumber isolates from other states, except for cucumber isolates from North Carolina, which showed moderate to great differentiation with Michigan cucumber isolates (Table

Table 8. Genetic clusters of isolates organized by whether they were retrieved from cucumber or another cucurbit host

\begin{tabular}{lrrrrrrrrr}
\hline & \multicolumn{8}{c}{ Number of isolates in predominate genetic cluster (\%) $^{\mathbf{a}}$} \\
\cline { 2 - 9 } Host & $\mathbf{1}$ & $\mathbf{2}$ & $\mathbf{3}$ & $\mathbf{4}$ & $\mathbf{5}$ & $\mathbf{6}$ & Mixed & Other & Total \\
\hline Cucumber & $42(88)$ & $10(77)$ & $29(81)$ & $6(86)$ & $15(15)$ & $40(85)$ & $182(91)$ & $10(83)$ & 334 \\
Other hosts & $6(12)$ & $3(23)$ & $7(19)$ & $1(14)$ & $87(85)$ & $7(15)$ & $18(9)$ & $2(17)$ & 131 \\
\hline
\end{tabular}

${ }^{a}$ Each isolate was assigned to the $K$ th genetic cluster to which the isolate predominately belonged based on results of Bayesian clustering. Mixed isolates were assigned partial membership in clusters 2,3 , and 4 . An isolate was assumed to belong equally to two or more genetic clusters if its membership was not $>0.1$ (proportion membership) higher in one genetic cluster than another; these isolates are designated "other."

Table 9. Genetic differentiation estimates of mitochondrial and nuclear genes for Pseudoperonospora cubensis isolates grouped by host

\begin{tabular}{|c|c|c|c|c|c|c|c|c|c|c|c|c|}
\hline \multirow[b]{4}{*}{ Category $^{b}$} & \multicolumn{12}{|c|}{$\mathbf{F}_{\mathbf{S T}}{ }^{\mathbf{a}}$} \\
\hline & \multirow{2}{*}{\multicolumn{2}{|c|}{$\begin{array}{c}\text { Host group } \\
\text { Cucumis spp. }\end{array}$}} & \multirow{2}{*}{\multicolumn{2}{|c|}{$\begin{array}{c}\text { Host type } \\
\text { Cucumber } \\
\end{array}$}} & \multicolumn{8}{|c|}{ Host species } \\
\hline & & & & & \multicolumn{2}{|c|}{ Cucumis melo } & \multicolumn{2}{|c|}{ C. sativus } & \multicolumn{2}{|c|}{ Cucurbita maxima } & \multicolumn{2}{|c|}{ C. moschata } \\
\hline & $\mathbf{M}$ & $\mathbf{N}$ & $\mathbf{M}$ & $\mathbf{N}$ & $\mathbf{M}$ & $\mathbf{N}$ & $\mathbf{M}$ & $\mathbf{N}$ & M & $\mathbf{N}$ & $\mathbf{M}$ & $\mathbf{N}$ \\
\hline \multicolumn{13}{|l|}{ Host group } \\
\hline $\begin{array}{l}\text { Cucurbita spp. } \\
\text { Host type }\end{array}$ & $0.52 *$ & $0.07^{*}$ & $\ldots$ & $\ldots$ & $\cdots$ & $\cdots$ & $\cdots$ & $\cdots$ & $\cdots$ & $\cdots$ & $\cdots$ & $\cdots$ \\
\hline \multicolumn{13}{|l|}{ Host species } \\
\hline Cucumis sativus & $\ldots$ & $\ldots$ & $\ldots$ & $\ldots$ & $0.31 *$ & $0.05 *$ & $\ldots$ & $\ldots$ & $\ldots$ & $\ldots$ & $\ldots$ & $\ldots$ \\
\hline Cucurbita maxima & $\ldots$ & $\ldots$ & $\ldots$ & $\ldots$ & 0.03 & $0.07 *$ & $0.43^{*}$ & $0.05^{*}$ & $\ldots$ & $\ldots$ & $\ldots$ & $\ldots$ \\
\hline C. moschata & $\ldots$ & $\ldots$ & $\ldots$ & $\ldots$ & $0.43^{*}$ & $0.15^{*}$ & $0.85 *$ & $0.23^{*}$ & $0.21 *$ & $0.21 *$ & $\ldots$ & $\ldots$ \\
\hline C. pepo & $\ldots$ & $\ldots$ & $\ldots$ & $\ldots$ & $0.14 *$ & $0.04 *$ & $0.64 *$ & $0.09 *$ & 0.02 & $0.05^{*}$ & $0.14 *$ & $0.07 *$ \\
\hline
\end{tabular}

${ }^{\text {a }}$ Average values for mitochondrial $(\mathrm{M})$ and nuclear genes $(\mathrm{N})$ are presented. ${ }^{*}$, significant at 0.05 .

${ }^{\mathrm{b}}$ Categories with less than eight isolates were excluded from analyses and are not shown. 
11). Michigan cucumber isolates showed moderate to great differentiation with non-cucumber isolates collected from other states (Table 11). When isolates collected from non-cucumber hosts were compared, moderate to high differentiation was observed between states, except for South Carolina and Texas, where low differentiation was suggested (Table 12). As noted above, diversity estimates tend to be higher for isolates from non-cucumber hosts than isolates from cucumber hosts. The highest diversity estimates were for non-cucumber isolates from Florida and North Carolina (Table 13).

P. cubensis temporal population structure. No clear structure was identified when isolates were grouped by year (Fig. 3C). For the 2007 to 2009 samples from the U.S., it was observed that clusters were similarly represented across the 3 years of sampling. Most samples from before 2004 were obtained from Europe, except for one sample from North Carolina that was obtained in 1982, which was a mixed isolate.

\section{Discussion}

This study investigated the genetic structure of $P$. cubensis populations from a worldwide collection. When Bayesian clustering was used to identify population subdivision, six genetic clusters were detected. Approximately half of the isolates belonged predominantly to one of five genetic clusters and the other half were mixed isolates (assigned approximately equal membership in clus-

Table 10. Diversity estimates of mitochondrial and nuclear genes for Pseudoperonospora cubensis isolates grouped by host

\begin{tabular}{|c|c|c|c|c|c|c|c|c|c|}
\hline \multirow[b]{3}{*}{ Category $^{b}$} & \multirow[b]{3}{*}{ Isolates $^{c}$} & \multicolumn{8}{|c|}{ Diversity estimates $^{\mathbf{a}}$} \\
\hline & & \multicolumn{2}{|c|}{ Hd } & \multicolumn{2}{|c|}{$\pi$} & \multicolumn{2}{|c|}{$\theta w$} & \multicolumn{2}{|c|}{$\mathbf{k}$} \\
\hline & & M & $\mathbf{N}$ & M & $\mathbf{N}$ & $\mathbf{M}$ & $\mathbf{N}$ & $\mathbf{M}$ & $\mathbf{N}$ \\
\hline \multicolumn{10}{|l|}{ Host group } \\
\hline Сucumis spp. & 391 & 0.28 & 0.28 & 0.001 & 0.001 & 0.23 & 1.27 & 0.30 & 0.53 \\
\hline Cucurbita spp. & 74 & 0.45 & 0.32 & 0.002 & 0.001 & 0.41 & 1.11 & 0.51 & 0.56 \\
\hline \multicolumn{10}{|l|}{ Host } \\
\hline Cucumis sativus & & 0.19 & 0.28 & 0.001 & 0.001 & 0.24 & 1.24 & 0.20 & 0.54 \\
\hline Other & 131 & 0.54 & 0.31 & 0.002 & 0.002 & 0.37 & 1.17 & 0.60 & 0.50 \\
\hline \multicolumn{10}{|l|}{ Host species } \\
\hline C. melo & 57 & 0.56 & 0.29 & 0.002 & 0.002 & 0.33 & 0.45 & 0.62 & 0.40 \\
\hline C. sativus & 334 & 0.19 & 0.30 & 0.001 & 0.002 & 0.24 & 1.24 & 0.20 & 0.58 \\
\hline Cucurbita maxima & 24 & 0.61 & 0.33 & 0.002 & 0.001 & 0.54 & 0.41 & 0.75 & 0.44 \\
\hline C. moschata & 12 & 0.15 & 0.12 & 0 & 0 & 0.17 & 0.11 & 0.15 & 0.14 \\
\hline С. реро & 38 & 0.40 & 0.32 & 0.001 & 0.002 & 0.36 & 1.18 & 0.43 & 0.68 \\
\hline
\end{tabular}

a Average values for mitochondrial $(\mathrm{M})$ and nuclear $(\mathrm{N})$ genes are presented. Hd, haplotype diversity; $\pi$, nucleotide diversity; $\theta \mathrm{w}$, Watterson's theta estimator per gene from sequence; $\mathrm{k}$, average number of nucleotide differences.

${ }^{\mathrm{b}}$ Categories with less than eight isolates were excluded from analyses and are not shown.

${ }^{c}$ Number of isolates.

Table 11. Genetic differentiation estimates of mitochondrial and nuclear genes for Pseudoperonospora cubensis isolates collected from cucumber (column) and non-cucumber (row) hosts grouped by U.S. state of origin

\begin{tabular}{|c|c|c|c|c|c|c|c|c|c|c|c|c|c|c|}
\hline \multirow[b]{3}{*}{ Category $^{\mathbf{b}}$} & \multicolumn{14}{|c|}{$\mathbf{F}_{\text {ST }}{ }^{\mathbf{a}}$} \\
\hline & \multicolumn{2}{|c|}{ Florida } & \multicolumn{2}{|c|}{ Georgia } & \multicolumn{2}{|c|}{ Louisiana } & \multicolumn{2}{|c|}{ Michigan } & \multicolumn{2}{|c|}{ North Carolina } & \multicolumn{2}{|c|}{ South Carolina } & \multicolumn{2}{|c|}{ Texas } \\
\hline & M & $\mathbf{N}$ & $\mathbf{M}$ & $\mathbf{N}$ & M & $\mathbf{N}$ & $\mathbf{M}$ & $\mathbf{N}$ & $\mathbf{M}$ & $\mathbf{N}$ & $\mathbf{M}$ & $\mathbf{N}$ & M & $\mathbf{N}$ \\
\hline California & $0.32 *$ & $0.06 *$ & $0.76^{*}$ & $0.11^{*}$ & $0.97 *$ & $0.09 *$ & 0 & 0.01 & $0.64 *$ & $0.11 *$ & $0.90 *$ & $0.20 *$ & $0.93 *$ & $0.19 *$ \\
\hline Delaware & 0.16 & 0.02 & $0.66^{*}$ & $0.08 *$ & $0.89 *$ & $0.11 *$ & 0 & $0.06^{*}$ & $0.52 *$ & $0.09 *$ & $0.82 *$ & $0.13 *$ & $0.84 *$ & $0.12 *$ \\
\hline Florida & $0.26^{*}$ & $0.03 *$ & $0.70 *$ & $0.10 *$ & $0.91 *$ & $0.11 *$ & 0.03 & $0.06^{*}$ & $0.57 *$ & $0.14 *$ & $0.85 *$ & $0.15^{*}$ & $0.87 *$ & $0.13^{*}$ \\
\hline Indiana & $0.30^{*}$ & $0.06 *$ & $0.73^{*}$ & $0.11 *$ & $0.94 *$ & $0.09 *$ & 0.01 & 0.02 & $0.60 *$ & $0.10^{*}$ & $0.88^{*}$ & $0.19 *$ & $0.90^{*}$ & $0.18 *$ \\
\hline Michigan & $0.28 *$ & $0.07 *$ & $0.79 *$ & $0.12 *$ & $1.00 *$ & $0.07 *$ & 0 & 0.05 & $0.66^{*}$ & $0.13^{*}$ & $0.93^{*}$ & $0.18 *$ & $0.95^{*}$ & $0.16^{*}$ \\
\hline North Carolina & 0 & $0.06 *$ & $0.36^{*}$ & $0.11 *$ & $0.60 *$ & 0.05 & $0.20 *$ & $0.07 *$ & $0.23^{*}$ & $0.05^{*}$ & $0.54 *$ & $0.21 *$ & $0.55^{*}$ & $0.20 *$ \\
\hline New York & $0.29 *$ & $0.05^{*}$ & $0.75^{*}$ & $0.11 *$ & $0.97 *$ & $0.10 *$ & 0 & $0.04 *$ & $0.62 *$ & $0.12 *$ & $0.90 *$ & $0.16^{*}$ & $0.92 *$ & $0.14 *$ \\
\hline Ohio & $0.31 *$ & $0.06 *$ & $0.74 *$ & $0.12 *$ & $0.95^{*}$ & $0.11 *$ & 0.01 & $0.03 *$ & $0.62 *$ & $0.15 *$ & $0.89 *$ & $0.17 *$ & $0.91 *$ & $0.16^{*}$ \\
\hline South Carolina & $0.34 *$ & $0.05^{*}$ & $0.79 *$ & $0.13^{*}$ & $1.00 *$ & $0.11 *$ & 0 & 0.03 & $0.66^{*}$ & $0.15^{*}$ & $0.93 *$ & $0.17 *$ & $0.95^{*}$ & $0.16^{*}$ \\
\hline Virginia & $0.30^{*}$ & $0.11 *$ & $0.71 *$ & $0.14 *$ & $0.92 *$ & $0.21 *$ & 0.06 & $0.14 *$ & $0.58^{*}$ & $0.19^{*}$ & $0.86^{*}$ & $0.18^{*}$ & $0.88^{*}$ & $0.17 *$ \\
\hline Wisconsin & $0.34 *$ & $0.07 *$ & $0.79 *$ & $0.13 *$ & $1.00 *$ & $0.12 *$ & 0 & 0.03 & $0.66^{*}$ & $0.14 *$ & $0.93^{*}$ & $0.18^{*}$ & $0.95^{*}$ & $0.16^{*}$ \\
\hline
\end{tabular}

a Average values for mitochondrial $(\mathrm{M})$ and nuclear genes $(\mathrm{N})$ are presented; * $=$ significant at 0.05 .

${ }^{\mathrm{b}}$ Categories with less than eight isolates were excluded from analyses and are not shown.

Table 12. Genetic differentiation estimates of mitochondrial and nuclear genes for Pseudoperonospora cubensis isolates from non-cucumber hosts grouped by U.S. state of origin

\begin{tabular}{|c|c|c|c|c|c|c|c|c|c|c|c|c|}
\hline \multirow[b]{3}{*}{ Category $^{\mathbf{b}}$} & \multicolumn{12}{|c|}{$\mathbf{F}_{\mathrm{ST}^{\mathrm{a}}}$} \\
\hline & \multicolumn{2}{|c|}{ Georgia } & \multicolumn{2}{|c|}{ Louisiana } & \multicolumn{2}{|c|}{ Michigan } & \multicolumn{2}{|c|}{ North Carolina } & \multicolumn{2}{|c|}{ South Carolina } & \multicolumn{2}{|c|}{ Texas } \\
\hline & $\mathbf{M}$ & $\mathbf{N}$ & $\mathbf{M}$ & $\mathbf{N}$ & $\mathbf{M}$ & $\mathbf{N}$ & M & $\mathbf{N}$ & $\mathbf{M}$ & $\mathbf{N}$ & M & $\mathbf{N}$ \\
\hline Florida & $0.22 *$ & 0 & $0.46^{*}$ & 0.05 & $0.27 *$ & $0.07 *$ & 0.10 & $0.04 *$ & $0.41 *$ & $0.05^{*}$ & $0.42 *$ & 0.03 \\
\hline Georgia & $\ldots$ & $\ldots$ & 0.18 & $0.08^{*}$ & $0.72 *$ & $0.12 *$ & 0 & $0.09 *$ & 0.05 & $0.05^{*}$ & 0.08 & $0.03 *$ \\
\hline Louisiana & $\ldots$ & $\ldots$ & $\ldots$ & $\ldots$ & $0.94 *$ & $0.11 *$ & $0.29 *$ & $0.10^{*}$ & 0.06 & $0.17 *$ & 0 & $0.15^{*}$ \\
\hline Michigan & $\ldots$ & $\ldots$ & $\ldots$ & $\ldots$ & $\ldots$ & $\ldots$ & $0.59 *$ & $0.15^{*}$ & $0.87 *$ & $0.21 *$ & $0.90 *$ & $0.19 *$ \\
\hline North Carolina & $\ldots$ & $\ldots$ & $\ldots$ & $\ldots$ & $\ldots$ & $\ldots$ & $\ldots$ & $\ldots$ & 0.16 & $0.15^{*}$ & 0.21 & $0.15^{*}$ \\
\hline South Carolina & $\ldots$ & $\ldots$ & $\ldots$ & $\ldots$ & $\ldots$ & $\ldots$ & $\ldots$ & $\ldots$ & $\ldots$ & $\ldots$ & 0 & $0.02 *$ \\
\hline
\end{tabular}

a Average values for mitochondrial $(\mathrm{M})$ and nuclear genes $(\mathrm{N})$ are presented; * $=$ significant at 0.05 .

${ }^{b}$ Categories with less than eight isolates were excluded from analyses and are not shown. 
ters 2, 3, and 4) according to genetic clustering. There was no direct correspondence between inferred genetic clusters and groupings of isolates by predefined geographic and host categories but some population structure was observed, with some clusters occurring more or less frequently in particular categories. Multilocus genotypes were shared across continental, host, and temporal scales, indicating that some genotypes are widely dispersed and persistent.

When isolates were grouped by geographic regions, all clusters were found in all continents; however, cluster 1 occurred more frequently in Europe, whereas clusters 2, 3, and 5 were more frequently found in North America. $\mathrm{F}_{\mathrm{ST}}$ values among isolates from North America, Europe, and Western Asia were low to moderate, which could be the result of infrequent human-mediated transport of $P$. cubensis, as also discussed by Runge et al. (50). $\mathrm{F}_{\mathrm{ST}}$ values are maintained at 0.1 or less, even if only a few individuals per generation migrate, resulting in gene flow between populations (19).

P. cubensis overwinters in southeast Europe $(24,27)$ and Western Asia, as well as in the southern part of North America, where cucurbits are produced year round. Sporangia of $P$. cubensis are wind dispersed and there is evidence of spread up to a thousand kilometers (40). Hence, within regions, limited genetic variation might be expected, and similar cluster composition was observed for countries in Western Europe. Because the pathogen can persist year round in southeast Europe, the high genetic diversity estimated for these countries (Greece and Italy) was expected. The high genetic diversity observed for isolates from the Czech Republic may reflect the more extensive collection of isolates included for this country but might also be influenced by the fact that all but five of these isolates originated from before 2004, in contrast to most other samples. A previous study based on phylogenetic analyses found that isolates from the Czech Republic, the Netherlands, and France were significantly different from isolates from Greece (54), in agreement with our findings.

Within the U.S., P. cubensis is hypothesized to spread from south to north along the East Coast (38). Limited genetic variation is expected between neighboring states because $P$. cubensis sporangia may be dispersed up to a thousand kilometers via wind currents (40). Ojiambo and Holmes (40) recently proposed that overwintering sources in southern Florida contribute directly to cucurbit downy mildew outbreaks from Florida up to North
Carolina. Surprisingly, isolates collected from Florida cucumber and non-cucumber hosts were moderately to highly differentiated from isolates from cucumber and non-cucumber hosts in North Carolina. Our study found that isolates from Georgia, North Carolina, Texas, South Carolina, and Indiana are genetically different from Florida, and high genetic diversity was detected in Georgia, Indiana, and North Carolina. These findings suggest that another source of inocula exists in addition to southern Florida, resulting in genetic differences and variability. This source could possibly be Mexico or southern states of the U.S. not included in our sampling. Oospores have not been observed in field samples from the U.S., and it is unclear if oospores play a role in the generation of genetic diversity and in pathogen overwintering in cucurbit crops or weeds. However, there are scattered reports of oospore formation in Europe (6), and abundant oospore formation under laboratory conditions was recently reported from Israel (10). The low genetic differentiation among isolates from Florida, South Carolina, and Virginia is consistent with Florida contributing inocula to states along the East Coast, in agreement with the findings of other studies (38-40). California had low diversity estimates; this may be due to its geographic separation from other states with severe downy mildew outbreaks. Isolates from Louisiana had a different population structure from isolates from other states, likely because of the low genetic and cluster diversity detected there.

It has been suggested that large-scale year-round production of cucumber in greenhouses in Ontario, Canada, may be a source of primary inoculum for field-grown cucumber in the Great Lakes states early in the growing season (40). The results of our study are consistent with this hypothesis, and similarities in cluster composition, low genetic differentiation, and comparable levels of genetic diversity were detected between isolates from the U.S. and Canada. Canadian isolates were obtained from Ontario, a province close to the Great Lakes states, and our analyses revealed high cluster similarity among Ontario, Michigan, and Ohio. $\mathrm{F}_{\mathrm{ST}}$ values indicate low to moderate genetic differentiation between isolates from Ontario and isolates from Michigan, Ohio, New York, Wisconsin, and Virginia. Moderate genetic differentiation was detected between isolates from Ontario and isolates from Delaware, Florida, Iowa, Indiana, North Carolina, South Carolina, and Texas, whereas high genetic differentiation was observed when Ontario isolates were compared pairwise with isolates from Georgia and Louisiana. Our results do not rule out the possibility that large-scale Ontario

Table 13. Diversity estimates of mitochondrial and nuclear genes for Pseudoperonospora cubensis isolates grouped by host (cucumber or other cucurbit host) and geography (U.S. state)

\begin{tabular}{|c|c|c|c|c|c|c|c|c|c|}
\hline \multirow[b]{3}{*}{ Category $^{\mathbf{b}}$} & \multirow[b]{3}{*}{ Isolates $^{c}$} & \multicolumn{8}{|c|}{ Diversity estimates $^{\text {a }}$} \\
\hline & & \multicolumn{2}{|c|}{ Hd } & \multicolumn{2}{|c|}{$\pi$} & \multicolumn{2}{|c|}{$\theta \mathbf{w}$} & \multicolumn{2}{|c|}{$\mathbf{k}$} \\
\hline & & $\mathbf{M}$ & $\mathbf{N}$ & $\mathbf{M}$ & $\mathbf{N}$ & $\mathbf{M}$ & $\mathbf{N}$ & $\mathbf{M}$ & $\mathbf{N}$ \\
\hline California cucumber & 30 & 0.07 & 0.15 & 0 & 0.001 & 0.15 & 0.10 & 0.07 & 0.15 \\
\hline Delaware cucumber & 20 & 0.20 & 0.17 & 0.001 & 0.001 & 0.35 & 0.11 & 0.20 & 0.17 \\
\hline Florida cucumber & 58 & 0.20 & 0.21 & 0.001 & 0.001 & 0.26 & 0.26 & 0.20 & 0.26 \\
\hline Florida other & 18 & 0.61 & 0.31 & 0.002 & 0.002 & 0.55 & 0.58 & 0.67 & 0.48 \\
\hline Georgia other & 32 & 0.43 & 0.29 & 0.001 & 0.002 & 0.45 & 0.30 & 0.45 & 0.42 \\
\hline Indiana cucumber & 24 & 0.15 & 0.18 & 0 & 0.001 & 0.17 & 0.21 & 0.15 & 0.20 \\
\hline Louisiana other & 18 & 0 & 0.31 & 0 & 0.003 & 0 & 0.35 & 0 & 0.36 \\
\hline Michigan cucumber & 60 & 0 & 0.20 & 0 & 0.001 & 0 & 0.43 & 0 & 0.36 \\
\hline Michigan other & 48 & 0.12 & 0.16 & 0 & 0.001 & 0.40 & 0.23 & 0.13 & 0.20 \\
\hline North Carolina cucumber & 26 & 0.53 & 0.21 & 0.002 & 0.001 & 0.48 & 0.16 & 0.56 & 0.26 \\
\hline North Carolina other & 20 & 0.60 & 0.32 & 0.002 & 0.001 & 0.53 & 0.39 & 0.69 & 0.41 \\
\hline New York cucumber & 38 & 0.05 & 0.13 & 0 & 0.001 & 0.14 & 0.14 & 0.05 & 0.14 \\
\hline Ohio cucumber & 46 & 0.12 & 0.12 & 0 & 0.001 & 0.14 & 0.18 & 0.12 & 0.17 \\
\hline South Carolina cucumber & 34 & 0 & 0.15 & 0 & 0.001 & 0 & 0.15 & 0 & 0.18 \\
\hline South Carolina other & 20 & 0.18 & 0.16 & 0 & 0.001 & 0.18 & 0.23 & 0.18 & 0.22 \\
\hline Texas other & 18 & 0.11 & 0.28 & 0 & 0.001 & 0.18 & 0.29 & 0.18 & 0.38 \\
\hline Virginia cucumber & 22 & 0.22 & 0.24 & 0.001 & 0.001 & 0.17 & 0.16 & 0.22 & 0.31 \\
\hline Wisconsin cucumber & 16 & 0 & 0.11 & 0 & 0.001 & 0 & 0.06 & 0 & 0.11 \\
\hline
\end{tabular}

a Average values for mitochondrial (M) and nuclear $(\mathrm{N})$ genes are presented. Hd, haplotype diversity; $\pi$, nucleotide diversity; $\theta w$, Watterson's theta estimator per gene from sequence; $\mathrm{k}$, average number of nucleotide differences.

${ }^{\mathrm{b}}$ Categories with less than eight isolates were excluded from analyses and are not shown.

c Number of isolates. 
greenhouses could be serving as a source of inoculum to some states in the Great Lakes region in addition to the inoculum received from states in the southern U.S. (40). Nonetheless, further studies that test for migration events of $P$. cubensis from Canada to the U.S. are needed to clarify the relative contributions of inocula to the Great Lakes states from possible sources in Ontario compared with possible sources in the southern U.S.

When isolates retrieved from $C$. sativus were compared with isolates from other cucurbit hosts, isolates from cluster 5 were noticeably more common in non-cucumber hosts. It is possible that isolates from different $P$. cubensis clusters are not equally adapted to the various cucurbit hosts and this could result in isolates from a specific cluster such as cluster 5 occurring more frequently in noncucumber hosts. In Israel, Cucurbita spp. were not hosts of $P$. $c u$ bensis until 2002 (9), when sporangia belonging to a pathotype capable of infecting Cucurbita spp. emigrated from southern or central Europe (24). More detailed P. cubensis population studies at the centers of origin or diversity of cucurbit hosts may clarify the differences observed in cluster occurrence in diverse hosts. Notably, in our study, diversity estimates were higher for isolates collected from non-cucumber hosts than from cucumber.

Our results indicated that mixed isolates were more frequently associated with $C$. sativus than with other cucurbit hosts. Recently (mid-2000s), resistance to $P$. cubensis introduced into cucumber became ineffective as a sole control mechanism for cucumber downy mildew in the U.S.. The presence of a new pathotype or physiological race in the U.S. has been proposed as an explanation for the breaking of resistance in cucumber by $P$. cubensis in approximately $2004(11,55,56)$. In addition, new pathotypes with an expanded host range have also been described in central Europe (25). A recent study (50) suggests that a new lineage of Pseudoperonospora might be responsible for current epidemics in the U.S.. Because mixed isolates were commonly associated with cucumber in different years of sampling in the U.S. (2007 to 2009), it is possible that the new pathotype, physiological race, species, or subspecies infecting cucumber is associated with mixed isolates. The historical sample from North Carolina (year 1982) was a mixed isolate, indicating that this type of isolate has been present in the U.S. since as early as 1982 . However, because we only had one U.S. sample obtained before approximately 2004, further studies, including herbarium specimens, are needed to confirm whether mixed isolates were common in the U.S. before approximately 2004.

This study provides an initial map of the population structure of $P$. cubensis. More clusters likely exist in $P$. cubensis because apparent structure may be underestimated when using sparse sampling and few markers (48). Previous studies have shown that, for clustering to be robust, less than 40 markers and as few as 7 markers (43) can be used if they are highly informative; however, using at least 50 markers is preferred (48). Future studies with uniform global sampling and hundreds of markers could determine whether additional genetic clusters are present in $P$. cubensis. The $P$. $\mathrm{cu}$ bensis genome (57), will provide an invaluable source of markers for robust population genetics studies. Nevertheless, the approach used in this study could be applied to other plant pathogens when using a large number of markers is not feasible due to high costs or scarce genomic resources, as long as markers used are informative. Continued genotyping of $P$. cubensis populations will be necessary to determine the existence of new clusters and to monitor the diversification of clusters detected in this study. A recent study showed that there is some limited infectivity of $P$. cubensis on hop and $P$. humuli on cucumber. In a multilocus phylogeny, Runge et al. (52) showed that $P$. humuli is separated and basal to P. cubensis, because they found that two lineages parasitic to hop species branched before the split of the two Pseudoperonospora lineages infecting Cucurbitaceae spp. This scenario suggested that an evolutionarily recent host jump from hops took place, with limited cross-infectivity potential present, because Runge and Thines (52) could infect hop with $P$. cubensis and cucumber with $P$. humuli, and confirm the result using molecular identification of emerging sporangiophores. Population structure analyses including $P$. humuli samples will be key to determine whether gene flow between these sister species exists or whether $P$. humuli populations contributed to the genetic variation observed in $P$. cubensis.

Breeding programs should include isolates that represent the genetic diversity of the pathogen in resistance screenings, given the high genetic variation found in this study and the previous finding that at least two distinct phylogenetic lineages are parasitic to Cucurbitaceae spp. (52). Isolates obtained from cucumber are commonly used in host resistance screenings but this study suggests that inclusion of isolates from C. melo and Cucurbita spp. that show a different genetic composition and high genetic diversity is necessary to capture the genetic variation of $P$. cubensis. In addition, regions with high genetic diversity such as the U.S. and Europe should be of special concern because $P$. cubensis populations with high levels of genetic variation are likely to adapt more rapidly to resistant hosts and fungicides than populations with little genetic variation. Gene pyramiding within a resistant variety or planting several resistant varieties in a field will be key to prevent or slow pathogen adaptation and future breaking of host resistance (4) by $P$. cubensis populations.

\section{Acknowledgments}

This material is based upon work supported by the Agricultural Research Fund, Pickle Packers International, Inc., Pickle and Pepper Research Committee of MSU, Pickle Packers International, Inc., Fresh Vegetable Growers of Ontario, North Central IPM Center Sub award 2003-51120-02111 S4256, Project GREEEN (a cooperative effort by Michigan plant-based commodities and businesses with the Michigan Agricultural Experiment Station, Michigan State University Extension and the Michigan Department of Agriculture) award numbers GR07-077 and GR06-0099D, the Ministry of Agriculture of the Czech Republic under project QH71229, the Ministry of Education, Youth and Sports of the Czech Republic under project MSM 6198959215, the Government of Hessen, Germany LOEWE funding program, and the Ministry of Science, Education and the Arts of Baden-Württemberg, Germany. We thank the many colleagues listed in Supplementary Table S1 for providing samples for this research; B. Day for giving us access to preliminary data of the P. cubensis genome project; all the members of the Hausbeck lab for their valuable suggestions and help; J. J. Morrice for providing the program Population Sorting Tool to generate population structure figures; and B. Webster, R. Heslip, A. Pianosi, H. Sweet, M. Wood, L. Rodriguez-Salamanca, and C. Erhardt for technical assistance.

\section{Literature Cited}

1. Altschul, S. F., Gish, W., Miller, W., Myers, E. W., and Lipman, D. J. 1990 Basic local alignment search tool. J. Mol. Biol. 215:403-410.

2. Altschul, S. F., Madden, T. L., Schaffer, A. A., Zhang, J., Zhang, Z., Miller, W., and Lipman, D. J. 1997. Gapped BLAST and PSI-BLAST: a new generation of protein database search programs. Nucleic Acids Res. 25:33893402 .

3. Bains, S. S., and Jhooty, J. S. 1976. Over wintering of Pseudoperonospora cubensis causing downy mildew of muskmelon. Indian Phytopathol. 29:213-214.

4. Barrett, L. G., Thrall, P. H., Burdon, J. J., and Linde, C. C. 2008. Life history determines genetic structure and evolutionary potential of host-parasite interactions. Trends Ecol. Evol. 23:678-685.

5. Baumgartner, K., Travadon, R., Bruhn, J., and Bergemann, S. E. 2010. Contrasting patterns of genetic diversity and population structure of Armillaria mellea sensu stricto in the eastern and western United States. Phytopathology 100:708-718.

6. Bedlan, G. 1989. Erstmaliger Nachweis von Oosporen von Pseudoperonospora cubensis (Berk. et Curt.) Rost. an Gewächshausgurken in Österreich. Pflanzenschutzberichte 50:119-120.

7. Blair, J. E., Coffey, M. D., Park, S.-Y., Geiser, D. M., and Kang, S. 2008. A multi-locus phylogeny for Phytophthora utilizing markers derived from complete genome sequences. Fungal Genet. Biol. 45:266-277.

8. Cohen, Y. 1981. Downy mildew of cucurbits. Pages 341-354 in: The Downy Mildews. D. M. Spencer, ed. Academic Press, London.

9. Cohen, Y., Meron, I., Mor, N., and Zuriel, S. 2003. A new pathotype of Pseudoperonospora cubensis causing downy mildew in cucurbits in Israel. Phytoparasitica 31:458-466.

10. Cohen, Y., Rubin, A. E., and Galperin, M. 2011. Formation and infectivity of oospores of Pseudoperonospora cubensis, the causal agent of downy mildew in cucurbits. Plant Dis. 95:874.

11. Colucci, S. J., and Holmes, G. J. 2007. Fungicide insensitivity and pathotype determination of Pseudoperonospora cubensis, causal agent of cucurbit downy mildew (Abstr.). Phytopathology 97:S24.

12. Cooke, D. E. L., Drenth, A., Duncan, J. M., Wagels, G., and Brasier, C. M. 2000. A molecular phylogeny of Phytophthora and related oomycetes. Fun- 
gal Genet. Biol. 30:17-32.

13. Donahoo, R. S., and Lamour, K. H. 2008. Interspecific hybridization and apomixis between Phytophthora capsici and Phytophthora tropicalis. Mycologia 100:911-920.

14. Doran, W. L. 1932. Downy mildew of cucumbers. Mass. Agric. Exp. Stn. Bull. 283.

15. Fu, Y. X., and Li, W. H. 1993. Statistical tests of neutrality of mutations. Genetics 133:693-709.

16. Gale, L. R., Ward, T. J., Balmas, V., and Kistler, H. C. 2007. Population subdivision of Fusarium graminearum sensu stricto in the upper midwestern United States. Phytopathology 97:1434-1439.

17. Gomez-Alpizar, L., Carbone, I., and Ristaino, J. B. 2007. An Andean origin of Phytophthora infestans inferred from mitochondrial and nuclear gene genealogies. Proc. Natl. Acad. Sci. USA 104:3306-3311.

18. Goss, E. M., Larsen, M., Chastagner, G. A., Givens, D. R., and Grunwald, N. J. 2009. Population genetic analysis infers migration pathways of Phytophthora ramorum in US nurseries. PLoS Pathog. 5:e10000583.

19. Hartl, D. L., and Clark, A. G. 1997. Principles of Population Genetics. Sinauer Associates, Inc., Sunderland, MA.

20. Holmes, G. J., Main, C. E., and Keever, Z. T. 2006. Cucurbit downy mildew: a unique pathosystem for disease forecasting. In: Advances in Downy Mildew Research. P. T. N. Spencer-Phillips and M. Jeger, eds. Kluwer Academic Publishers, Dordrecht, The Netherlands.

21. Holmes, G. J., and Thomas, C. E. 2006. The history and re-emergence of cucurbit downy mildew (Abstr.). Phytopathology 99:S171.

22. Hudson, R. R. 1987. Estimating the recombination parameter of a finite population model without selection. Genet. Res. 50:245-250.

23. Hudson, R. R., and Kaplan, N. L. 1985. Statistical properties of the number of recombination events in the history of a sample of DNA sequences. Genetics 111:147-164.

24. Lebeda, A., and Cohen, Y. 2011. Cucurbit downy mildew (Pseudoperonospora cubensis) — biology, ecology, epidemiology, host-pathogen interaction and control. Eur. J. Plant Pathol. 129:157-192.

25. Lebeda, A., Hubschova, J., and Urban, J. 2010. Temporal population dynamics of Pseudoperonospora cubensis. In: Proceedings of Cucurbitaceae 2010. J. A. Thies, S. Kousik and A. Levi, eds. Universal Press, Alexandria, VA.

26. Lebeda, A., Pavelkova, J., Urban, J., and Sedlakova, B. 2011. Distribution, host range and disease severity of Pseudoperonospora cubensis on cucurbits in the Czech Republic. J. Phytopathol. 159:589-596.

27. Lebeda, A., and Schwinn, F. J. 1994. The downy mildews - an overview of recent research progress. J. Plant Dis. Prot. 101:225-254.

28. Lebeda, A., and Urban, J. 2007. Temporal changes in pathogenicity and fungicide resistance in Pseudoperonospora cubensis populations. Acta Hortic. 731:327-336.

29. Lebeda, A., and Widrlechner, M. P. 2004. Response of wild and weedy Cucurbita L. to pathotypes of Pseudoperonospora cubensis (Berk. \& Curt.) Rostov. (cucurbit downy mildew). Pages 203-210 in: Advances in Downy Mildew Research. P. T. N. Spencer-Phillips and M. Jeger, eds. Kluwer Academic Publishers, Dordrecht, The Netherlands.

30. Librado, P., and Rozas, J. 2009. DnaSP v5: a software for comprehensive analysis of DNA polymorphism data. Bioinformatics 25:1451-1452.

31. Maddison, W. P., and Maddison, D. R. 2002. MacClade: Analysis of Phylogeny and Character Evolution, 4.06 OSX. Sunderland, MA

32. Maniatis, T., Fritsch, E. F., and Sambrook, J. 1982. Molecular Cloning: A Laboratory Manual. Cold Spring Harbor Laboratory, Cold Spring Harbor, NY.

33. McGinnis, S., and Madden, T. L. 2004. BLAST: at the core of a powerful and diverse set of sequence analysis tools. Nucleic Acids. Res. 32:W20W25.

34. Mitani, S., Araki, S., Yamaguchi, T., Takii, Y., Ohshima, T., and Matsuo, N. 2001. Biological properties of the novel fungicide cyazofamid against Phytophthora infestans on tomato and Pseudoperonospora cubensis on cucumber. Pest Manage. Sci. 58:139-145.

35. Moncalvo, J. M., Wang, H. H., and Hseu, R. S. 1995. Phylogenetic relationships in Ganoderma inferred from the internal transcribed spacers and $28 \mathrm{~S}$ ribosomal DNA sequences. Mycologia 87:223-223.

36. Moss, M. A. 1987. Resistance to metalaxyl in the Pseudoperonospora cubensis population causing downy mildew of cucumber in south Florida. Plant Dis. 71:1045.

37. Nei, M. 1987. Molecular Evolutionary Genetics. Columbia University Press, New York

38. Nusbaum, C. J. 1944. The seasonal spread and development of cucurbit downy mildew in the Atlantic coastal states. Plant Dis. 28:82-85.

39. Nusbaum, C. J. 1948. A summary of cucurbit downy mildew reports from Atlantic coastal states in 1947. Plant Dis. 32:44-48.

40. Ojiambo, P., and Holmes, G. 2011. Spatio-temporal spread of cucurbit downy mildew in the eastern United States. Phytopathology 101:451-461.
41. Palti, J., and Cohen, Y. 1980. Downy mildew of cucurbits (Pseudoperonospora cubensis): the fungus and its hosts, distribution, epidemiology, and control. Phytoparasitica 8:109-147.

42. Park, J., Park, B., Veeraraghavan, N., Jung, K., Lee, Y.-H., Blair, J. E., Geiser, D. M., Isard, S., Mansfield, M. A., Nikolaeva, E., Park, S.-Y., Russo, J., Kim, S., H., Greene, M., Ivors, K. L., Balci, Y., Peiman, M., Erwin, D. C., Coffey, M. D., Rossman, A., Farr, D., Cline, E., Grunwald, N. J., Luster, D. G., Schrandt, J., Martin, F., Ribeiro, O. K., Makalowska, I., and Kang, S. 2008. Phytophthora database: a forensic database supporting the identification and monitoring of Phytophthora. Plant Dis. 92:966-972.

43. Pritchard, J. K., Stephens, M., and Donnelly, P. 2000. Inference of population structure using multilocus genotypic data. Genetics 155:945-959.

44. Quesada-Ocampo, L. M., Granke, L. L., Mercier, M. R., Olsen, J., and Hausbeck, M. K. 2011. Investigating the genetic structure of Phytophthora capsici populations. Phytopathology 101:1061-1073.

45. R-Development-Core-Team. 2008. R: A Language and Environment for Statistical Computing. R Foundation for Statistical Computing, Vienna

46. Reuveni, M., Eyal, H., and Cohen, Y. 1980. Development of resistance to Metalaxyl in Pseudoperonospora cubensis. Plant Dis. 64:1108-1108.

47. Riethmuller, A., Voglmayr, H., Goker, M., Weib, M., and Oberwinkler, F. 2002. Phylogenetic relationships of the downy mildews (Peronosporales) and related groups based on nuclear large subunit ribosomal DNA sequences. Mycologia 94:834-849.

48. Rosenberg, N. A., Mahajan, S., Ramachandran, S., Zhao, C., Pritchard, J. K., and Feldman, M. W. 2005. Clines, clusters, and the effect of study design on the inference of human population structure. PLoS Genet. 1:e70.

49. Rozen, S., and Skaletsky, H. J. 2000. Primer3 on the WWW for General Users and for Biologist Programmers. Humana Press, Totowa, NJ.

50. Runge, F., Choi, Y.-J., and Thines, M. 2011. Phylogenetic investigations in the genus Pseudoperonospora reveal overlooked species and cryptic diversity in the P. cubensis cluster. Eur. J. Plant Pathol. 129:135-146.

51. Runge, F., and Thines, M. 2009. A potential perennial host for Pseudoperonospora cubensis in temperate regions. Eur. J. Plant Pathol. 123:483486.

52. Runge, F., and Thines, M. 2012. Re-evaluation of host specificity of the closely related species Pseudoperonospora humuli and P. cubensis. Plant Dis. 96:55-61.

53. Samoucha, Y., and Cohen, Y. 1984. Synergy between metalaxyl-sensitive and metalaxyl-resistant strains of Pseudoperonospora cubensis. Phytopathology 74:376-378.

54. Sarris, P., Abdelhalim, M., Kitner, M., Skandalis, N., Panopoulos, N., Doulis, A., and Lebeda, A. 2009. Molecular polymorphisms between populations of Pseudoperonospora cubensis from Greece and the Czech Republic and the phytopathological and phylogenetic implications. Plant Pathol. 58:933-943.

55. Savory, E. A., Granke, L. L., Quesada-Ocampo, L. M., Varbanova, M., Hausbeck, M. K., and Day, B. 2011. The cucurbit downy mildew pathogen Pseudoperonospora cubensis. Mol. Plant Pathol. 12:217-226.

56. Savory, E. A., Tian, M., Erhardt, C., Hausbeck, M., Hammerschmidt, R., and Day, B. 2008. An integrative approach to characterizing the cucumberPseudoperonospora cubensis interaction. (Abstr.) Phytopathology 98:S140.

57. Savory, E., Zou, C., Adhikari, B., Hamilton, J. P., Buell, C. R., Shiu, S.-H., and Day, B. 2012. Alternative splicing of a multi-drug transporter from Pseudoperonospora cubensis generates an RXLR effector protein that elicits a rapid cell death. PLoS ONE 7(4): e34701. doi:10.1371/journal. pone.0034701

58. Spencer, D. M. 1981. The Downy Mildews. Academic Press Inc., London.

59. Tajima, F. 1983. Evolutionary relationship of DNA sequences in finite populations. Genetics 105:437-460.

60. Tajima, F. 1989. Statistical method for testing the neutral mutation hypothesis by DNA polymorphism. Genetics 123:585-595.

61. Thomas, C. E. 1996. Downy Mildew. Pages 25-27 in: Compendium of Cucurbit Diseases. T. A. Zitter, D. L. Hopkins and C. E. Thomas, eds. American Phytopathological Society, St. Paul, MN.

62. Thomas, C. E., Inaba, T., and Cohen, Y. 1987. Physiological specialization in Pseudoperonospora cubensis. Phytopathology 77:1621-1624.

63. Urban, J., and Lebeda, A. 2006. Fungicide resistance in cucurbit downy mildew-methodological, biological and population aspects. Ann. Appl. Biol. 149:63-75.

64. Urban, J., and Lebeda, A. 2007. Variation of fungicide resistance in Czech populations of Pseudoperonospora cubensis. J. Phytopathol. 155:143-151.

65. Van Haltern, F. 1933. Spraying cantaloupes for the control of downy mildew and other diseases. Ga. Exp. Stn. Bull. 175.

66. Watterson, G. A. 1975. On the number of segregating sites in genetical models without recombination. Theor. Popul. Biol. 7:256-276.

67. Zhu, S., Liu, P., Liu, X., Li, J., Yuan, S., and Si, N. 2008. Assessing the risk of resistance in Pseudoperonospora cubensis to the fungicide flumorph in vitro. Pest Manage. Sci. 64:255-261. 